\title{
Evaluation and Bias Correction in WRF Model Forecasting of Precipitation and Potential Evapotranspiration
}

\author{
THEODOR BUGHICI AND NAFTALI LAZAROVITCH \\ Wyler Department of Dryland Agriculture, French Associates Institute for Agriculture and Biotechnology \\ of Drylands, Jacob Blaustein Institutes for Desert Research, Ben-Gurion University of \\ the Negev, Sede Boqer Campus, Israel \\ ERICK FREDJ \\ Jerusalem College of Technology, Department of Computer Science, Jerusalem, Israel \\ ERAN TAS \\ The Robert H. Smith Faculty of Agriculture, Food and Environment, Department of Soil \\ and Water Sciences, The Hebrew University of Jerusalem, Rehovot, Israel
}

(Manuscript received 31 July 2018, in final form 19 March 2019)

\begin{abstract}
A reliable forecast of potential evapotranspiration $\left(\mathrm{ET}_{0}\right)$ is key to precise irrigation scheduling toward reducing water and agrochemical use while optimizing crop yield. In this study, we examine the benefits of using the Weather Research and Forecasting (WRF) Model for $\mathrm{ET}_{0}$ and precipitation forecasts with simulations at a 3-km grid spatial resolution and an hourly temporal resolution output over Israel. The simulated parameters needed to calculate $\mathrm{ET}_{0}$ using the Penman-Monteith (PM) approach, as well as calculated $\mathrm{ET}_{0}$ and precipitation, were compared to observations from a network of meteorological stations. WRF forecasts of all PM meteorological parameters, except wind speed $W_{s}$, were significantly sensitive to seasonality and synoptic conditions, whereas forecasts of $W_{s}$ consistently showed high bias associated with strong local effects, leading to high bias in the evaluated $\mathrm{PM}-\mathrm{ET}_{0}$. Local $W_{s}$ bias correction using observations on days preceding the forecast and interpolation of the resulting $\mathrm{PM}-\mathrm{ET}_{0}$ to other locations led to significant improvement in $\mathrm{ET}_{0}$ forecasts over the investigated area. By using this hybrid forecast approach $\left(\mathrm{WRF}_{\mathrm{BC}}\right)$ that combines WRF numerical simulations with statistical bias corrections, daily $\mathrm{ET}_{0}$ forecast bias was reduced from an annual mean of $13 \%$ with $\mathrm{WRF}$ to $3 \%$ with $\mathrm{WRF}_{\mathrm{BC}}$, while maintaining a high model-observation correlation. WRF was successful in predicting precipitation events on a daily event basis for all four forecast lead days. Considering the benefit of the hybrid approach for forecasting $\mathrm{ET}_{0}$, the WRF Model was found to be a highpotential tool for improving crop irrigation management.
\end{abstract}

\section{Introduction}

Reliable evapotranspiration and precipitation predictions are essential for high-precision crop-irrigation scheduling. In the face of growing water scarcity and declining water quality on the one hand, and increasing food demand of the growing world population on the other, improving the efficiency of crop water use is of the utmost importance (Steduto et al. 2012). Efficient irrigation management should rely on precise medium-range (i.e., 3-7 days) forecasts of crop water

\footnotetext{
Corresponding author: Eran Tas, eran.tas@mail.huji.ac.il
}

requirements and precipitation resulting in minimized yield reduction, irrigation cost, nutrient leaching and pollution of surface and groundwater by agrochemicals (Ali 2010).

The Penman-Monteith (PM) equation is wellrecognized to be scientifically adequate for evaluating optimal crop water demand by calculating the potential evapotranspiration $\left(\mathrm{ET}_{0}\right)$ (e.g., López-Urrea et al. 2006; Kool et al. 2014). The protocol established by the Food and Agriculture Organization of the United Nations (FAO Irrigation and Drainage Paper 56; FAO-56) is widely used to adjust the PM equation for $\mathrm{ET}_{0}$ calculation to specific crop types and ambient conditions 
(Allen et al. 1998). The $\mathrm{ET}_{0}$ calculation as described in the FAO-56 protocol is based on air temperature $T$, solar radiation $R_{s}$, wind speed $W_{s}$, and air relative humidity (RH) data. Therefore, a reliable forecast of $\mathrm{ET}_{0}$ for precise irrigation should rely on accurate meteorological parameter forecasts at high temporal and spatial resolution.

Medium-range weather forecasts can be made by using either statistical models or numerical weather predictions (NWPs). Statistical models are generally not appropriate for medium-range precipitation forecasting, considering the complex manner in which both local and synoptic-driven precipitation depend on temporal variation of many variables. In addition a main disadvantage of statistical models for weather prediction is that they cannot be implemented in locations lacking previous long-term observations to provide a reliable statistical basis (Kumar et al. 2011), resulting in $\mathrm{ET}_{0}$ predictions that are not accurate enough for precise irrigation (e.g., Luo et al. 2015).

Early versions of NWP models used low spatial resolution to forecast PM parameters and $\mathrm{ET}_{0}$, and results exhibited low correlations and large errors (e.g., Duce et al. 1999; Arca et al. 2003). In recent years, however, the forecasting performance of mesoscale NWP models has improved significantly, encouraging their use to forecast weather variables, including precipitation, at high temporal and spatial resolutions for a variety of research and operational purposes (Bauer et al. 2015). Therefore, NWP models should be the preferred tool for irrigation decision-making.

Since its initial release in 2000, the Weather Research and Forecasting (WRF) Model (Skamarock et al. 2008) has become one of the world's most widely used NWP models, designed to serve both research and operational needs. It is a free, open-source, mature, and constantly advancing model (Powers et al. 2017). While efforts have been made to study the WRF Model's ability to predict $\mathrm{ET}_{0}$ and despite considerable progress in recent years, its forecast is still subject to biases that may preclude its direct use for agricultural purposes. The WRF Model's ability to accurately forecast surface variables may be sensitive to choice of parameterization (Ruiz et al. 2010; Ries and Schlünzen 2009; Zittis et al. 2014; Noble et al. 2014, 2017) and accuracy of land-use data and terrain representation (Santos-Alamillos et al. 2013; Jiménez-Esteve et al. 2018) and can also exhibit systematic error with respect to seasonality (e.g., Gunwani and Mohan 2017; García-Díez et al. 2013), synoptic pressure systems (Ilotoviz et al. 2015), and geographical location (e.g., Libonati et al. 2008). Moreover, the meteorological parameters required to evaluate $\mathrm{PM}-\mathrm{ET}_{0}$ are themselves interdependent and can either enhance or cancel out errors (e.g., WRF forecast RH bias caused by $T$ bias; Cheng and Steenburgh 2005).

In an ensemble of high-resolution WRF simulations over Europe and the Mediterranean area, Kioutsioukis et al. (2016) found that at the farm scale $\left(2 \times 2 \mathrm{~km}^{2}\right), T$ is well simulated, $\mathrm{RH}$ is underestimated at most sites, $W_{s}$ and $\mathrm{ET}_{0}$ are overestimated, and precipitation forecast skill was highly variable across latitude, season, and parameterization schemes. Forecast errors of $W_{s}$ are a recurring issue in NWP models, WRF in particular, where it is usually over predicted (Cheng and Steenburgh 2005; Srivastava et al. 2015; Kioutsioukis et al. 2016) to the point of being the primary cause of $\mathrm{ET}_{0}$ error (Perera et al. 2014). The $W_{s}$ errors with the WRF Model are usually hard to resolve since they are attributed to misrepresentation of local terrain and the associated turbulent drag (Jiménez and Dudhia 2012; SantosAlamillos et al. 2013).

Reliable precipitation predictions can also improve irrigation management by delaying irrigation when rain events are expected, thereby saving water and nutrients, and preventing excess agrochemical leaching and oxygen deficiencies in saturated soils (Bhattarai et al. 2010). In terms of irrigation decision-making, NWP forecast analysis of precipitation can be better approached for the probability prediction of a daily rainfall event, rather than an accurate prediction of quantities (e.g., Wang and Cai 2009).

There are available methods that can be applied for improving WRF forecast accuracy and reducing biases, including data-assimilation techniques (e.g., Wang et al. 2014) and predictor-corrector methods that can be applied at the postprocessing stage, such as the Kalman filter (e.g., Diagne et al. 2014). However, these methods require a high level of expertise and extensive observational data that are rarely available, currently making them hard to implement, and are not yet established in our region at present. When forecast bias is systematic, prediction error can be reduced based on past errors. Hacker and Rife (2007) were able to reduce nearsurface 6-h forecast temperatures based on observed running mean errors, but could not correct $W_{0}$ bias due to low systematic forecast errors.

The objectives of our study were to (i) investigate the performance of PM-based $\mathrm{ET}_{0}$ and precipitation predictions by the WRF Model for crop-irrigation practice in Israel and their dependence on seasonality, geography, and synoptic pressure regimes, and (ii) test a biascorrection scheme, which is based on hybridization of observations and WRF modeling. The studied area is particularly attractive for its high diversity, with sharp climatic and topographic gradients. We examined the WRF forecast over 1 year against observations from 
29 weather stations. We show that the hybrid corrected model maintains the high correlation of daily $\mathrm{ET}_{0}$ with observations and yet reduces forecast bias for any point of interest so that higher irrigation precision could be achieved.

\section{Methodology}

\section{a. Study area}

The study area used in this work includes the entire area of Israel, located between the eastern Mediterranean and the Red Sea (see Fig. 1). The Mediterranean region forms a transitional zone between the midlatitudes of western and central Europe and the arid regions of North Africa and the Middle East, with the inland Mediterranean Sea in between. Israel is located in the eastern part of the Mediterranean, influenced by westerlies in the wet winter season (October-May) and the blocking subtropical high during the dry summer season (June-September). Extreme climatic northsouth and west-east gradients are typical for this region, as is topographic heterogeneity. The southern part of Israel belongs to the desert belt extending from the Sahara through Egypt and Sinai to the Arabian Desert. Roughly the southern half (south to $31.2^{\circ} \mathrm{N}$ ) of Israel is semiarid and arid $\left(<250 \mathrm{~mm} \mathrm{yr}^{-1}\right.$, mainly the Negev and Arava Valley areas, see Fig. 1), while the northern part has a Mediterranean climate with a dry summer and cool, wet winter (mainly the Galilee and Coastal Plain areas, see Fig. 1). The topography consists of three strips running south (or southwest) to north. From west to east, respectively, these strips are the Coastal Plain, the hilly region, the Jordan Valley and the Arava Valley, spanning from the Dead Sea to the Red Sea. The study area is small (on the order of $100 \mathrm{~km} \times 400 \mathrm{~km}$ ) and has intensive agricultural production marked by continuous striving for higher efficiency; it is an attractive case study due to strong climatic and topographic gradients. Therefore, a reliable weather forecast for our study area should be of high spatial and temporal resolution, and able to downscale agro-meteorological parameters to different climatic regions that are several kilometers apart. The research was conducted for the year 2013, an overall average year apart from an extreme cold weather event for 4 days in mid-December.

\section{b. WRF Model setup}

Simulations were performed using the nonhydrostatic Advanced Research version of WRF (WRF-ARW) mesoscale model (version 3.4). The simulations were driven using the National Centers for Environmental Prediction Global Forecast System (GFS). The GFS spatial resolution was $1^{\circ} \times 1^{\circ}$ and with 6 -h time resolution.
The model configuration with two-way interacting domains included three successive nested domains, with grid-spacing of $27 \mathrm{~km}$ (domain 1), $9 \mathrm{~km}$ (domain 2), and $3 \mathrm{~km}$ (domain 3 ) and 27 vertical levels of the atmospheric data (Fig. 1). The forecast term ranged from 0 to 96 lead hours, starting at 0000 UTC daily with hourly data output, for each day of the year. The model was operated with the WRF single-moment 6-class microphysics scheme (WSM6; Hong and Lim 2006), the Mellor-Yamada-Janjić planetary boundary layer scheme, the Kain-Fritsch cumulus scheme (Fritsch and Kain 1993), the unified Noah land surface model, the RRTM scheme for longwave radiation (Mlawer et al. 1997), and the MM5 shortwave scheme of Dudhia (1989) for shortwave radiation. Between domains 1 and 2, the two-way nesting option was selected to allow interaction of grids in both directions. Note that 2-m zonal and meridional winds ( $U$ and $V$, respectively) were calculated based on the $10-\mathrm{m}$ values using a logarithmic vertical profile-based approach, and $U$ and $V$ were used to calculate $W_{s}$. RH at $2 \mathrm{~m}$ was calculated using the 2-m specific humidity $q$. Eventually, 2-m $T, R_{s}, \mathrm{RH}$, and $W_{s}$ were used for $\mathrm{ET}_{0}$ calculation (as described in section 2c). Note that in addition to WRF, two observation-based prediction methods were used in this study (sections 2e and 2f), and the three methods are summarized in Table 1.

\section{c. Potential evapotranspiration calculation}

The presented analyses relies on evaluation of $\mathrm{ET}_{0}$ using both observations by meteorological stations and model simulations using WRF. In both cases, $\mathrm{ET}_{0}$ was evaluated based on PM using the well-accepted FAO-56 protocol, which provides a highly practical and widely used approach to evaluating $\mathrm{ET}_{0}$ based on the PM equation (Allen et al. 1998). According to this protocol the operative equation for calculating the evapotranspiration of well-watered grass is

$$
\mathrm{ET}_{0}=\frac{0.408 \Delta\left(R_{n}-G\right)+\gamma \frac{900}{T+273} W_{s}\left(e_{s}-e_{a}\right)}{\Delta+\gamma\left(1+0.34 W_{s}\right)},
$$

where $T$ is the air temperature at a height of $2 \mathrm{~m}\left({ }^{\circ} \mathrm{C}\right) ; R_{n}$ is the net radiation at the crop surface $\left(\mathrm{MJ} \mathrm{m}^{-2}\right.$ day $\left.^{-1}\right)$; $G$ is the soil heat flux $\left(\mathrm{MJ} \mathrm{m}^{-2} \mathrm{day}^{-1}\right) ; e_{s}$ and $e_{a}$ are the saturated and air vapor pressure, respectively $(\mathrm{kPa}) ; \gamma$ is the psychometric constant $\left(\mathrm{kPa}^{\circ} \mathrm{C}^{-1}\right) ; \Delta$ is the slope of saturation vapor pressure versus air temperature curve $\left(\mathrm{kPa}^{\circ} \mathrm{C}^{-1}\right)$; and $W_{s}$ is the 2-m height wind speed $\left(\mathrm{m} \mathrm{s}^{-1}\right)$. We used $R_{s}$ and RH to calculated $R_{n}$ and $e_{a}$, respectively, according to the procedures described in the FAO-56 protocol. The daily $\mathrm{ET}_{0}$ values were obtained 

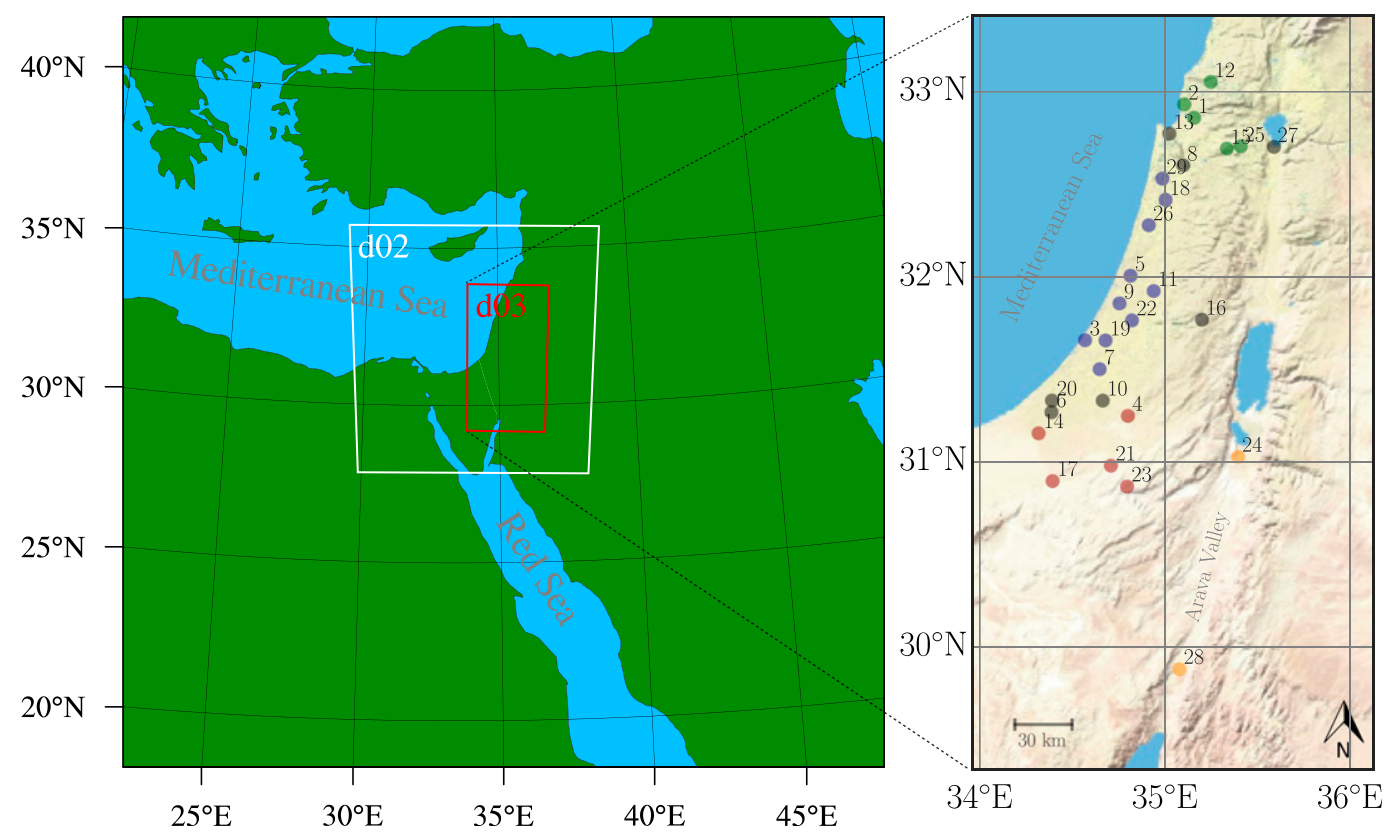

FIG. 1. (left) WRF Model computational domains of the simulation including three successive nested domains, with grid spacing of 27, 9, and $3 \mathrm{~km}$. (right) Domain 3 (d03) is enlarged with numbered locations of the meteorological observational stations. Locations in the Galilee area, Coastal Plain, Negev Desert, and Arava Valley are marked in green, purple, red, and orange, respectively.

by integrating hourly $\mathrm{ET}_{0}$, using the cubic spline interpolation method in cases of missing values of up to $2 \mathrm{~h}$, a method commonly used to fill in missing weather data (e.g., Levermore and Parkinson 2006).

\section{d. Observational data and validation methods}

Observations from 29 standard meteorological stations (provided by the Israeli Meteorological Service; https://ims.data.gov.il/) were used for model evaluation. All stations were located within the area represented by the innermost domain (domain 3, see Fig. 1). Simulated data for each of the WRF grid points were tested against the observed data of the nearest station. The meteorological stations did not measure $q$ nor air pressure, which are WRF Model prognostic variables. We have evaluated $q$ values using measured $\mathrm{RH}, T$, and the standard air pressure at station altitude, neglecting possible large deviations for the latter. Thus, $q$ validation was only used as supporting data for $\mathrm{RH}$ validation.
WRF was evaluated for its ability to predict each of the PM variables and $\mathrm{PM}-\mathrm{ET}_{0}$ and daily rain events. The latter was performed using the receiver operating characteristic (ROC) curve. A daily precipitation event can be regarded as dichotomous based on a predetermined threshold (Jolliffe and Stephenson 2012) and therefore can be regarded as a simple binary approach. The ROC score was evaluated based on the area under the ROC curve (AUC), taken as a scalar measure (Hanley and McNeil 1982), with an AUC of 0.5 reflecting random predictions and AUC $=1$ implying a perfect prediction. A threshold of $5 \mathrm{~mm}$ was applied to define daily rain occurrence. Four statistical approaches were used to evaluate $\mathrm{PM}$ variables and $\mathrm{PM}-\mathrm{ET}_{0}$ forecast quality: the index of agreement (IOA; Willmott 1981), mean bias, mean fractional bias (FB), and root-meansquare error (RMSE). We found IOA to be important for the forecast evaluation, as it includes a correlation-based measure of the differences between

TABLE 1. The three forecast methods analyzed and compared for $\mathrm{ET}_{0}$ predictions.

\begin{tabular}{|c|c|c|}
\hline Method & Description & Classification \\
\hline WRF & WRF forecast model used for $\mathrm{ET}_{0}$ and rain forecasts (section $2 \mathrm{~b}$ ) & WRF forecast \\
\hline $\mathrm{WRF}_{\mathrm{BC}}$ & $\begin{array}{l}\text { WRF forecast model after } W_{s} \text { bias correction, } \mathrm{ET}_{0} \text { calculation, and interpolation } \\
\text { (section } 2 \mathrm{e} \text { ). This method was used in order to improve } \mathrm{WRF} \mathrm{ET}_{0} \text { forecasts. }\end{array}$ & Hybrid WRF-observations \\
\hline IDW & $\begin{array}{l}\left.\mathrm{ET}_{0} \text { based on interpolated mean values of observations to forecast time (section } 2 \mathrm{f}\right) \text {. This } \\
\text { method was used to investigate } \mathrm{WRF} \text { and } \mathrm{WRF}_{\mathrm{BC}} \mathrm{ET}_{0} \text { forecast advantages. }\end{array}$ & Observations only \\
\hline
\end{tabular}


model predictions and observations in terms of the mean and variance:

$$
\mathrm{IOA}=1-\frac{\sum_{i=0}^{n}\left(P_{i}^{\mathrm{obs}}-P_{i}^{\text {model }}\right)^{2}}{\sum_{i=0}^{n}\left(\left|P_{i}^{\mathrm{model}}-\overline{P^{\mathrm{obs}}}\right|+\left|P_{i}^{\mathrm{obs}}-\overline{P^{\mathrm{obs}}}\right|\right)^{2}},
$$

where $n$ is the number of observations and $P_{i}^{\text {obs }}, \overline{P^{\text {obs }}}$, and $P_{i}^{\text {model }}$ are observed data as measured at the meteorological stations, their mean, and WRF Model data, respectively. IOA varies between 0 and 1 with a value of 1 indicating a perfect match, and 0 no agreement at all. The mean bias was calculated as

$$
\operatorname{bias}=\frac{\sum_{i=0}^{n}\left(P_{i}^{\text {model }}-P_{i}^{\text {obs }}\right)}{n} .
$$

FB is a dimensionless and normalized bias score. It varies between +2 (overprediction) and -2 (underprediction) and has a value of zero for an ideal model. It is given by

$$
\mathrm{FB}=\frac{2 \sum_{i=0}^{n}\left(P_{i}^{\text {model }}-P_{i}^{\mathrm{obs}}\right)}{\sum_{i=0}^{n}\left(P_{i}^{\mathrm{model}}+P_{i}^{\mathrm{obs}}\right)}
$$

The RMSE expresses average model prediction error in units of the variable of interest. The errors are squared before they are averaged, thus the RMSE gives a relatively high weight to large errors, given by

$$
\mathrm{RMSE}=\sqrt{\frac{\sum_{i=1}^{n}\left(P_{i}^{\text {model }}-P_{i}^{\mathrm{obs}}\right)^{2}}{n} .}
$$

Since a biased model can still result in relatively high IOA scores, the bias and RMSE indices are important as complementary statistical parameters. IOA and FB are normalized, unitless scores thus enabling us to compare the forecasts of parameters with different units, while the bias has the parameter's units and is used in this paper to evaluate daily $\mathrm{ET}_{0}$ predictions.

Model performance was tested with respect to lead days, location, season, and synoptic pressure system class. The seasonal features of the eastern Mediterranean are influenced by the big pressure systems of the Atlantic, Eurasia, and Africa, and therefore a division of the year into seasons can also be devised into characteristic synoptic types that are distinct from the seasonal types. Seven out of the 18 synoptic pressure system classes
(Table 2) that were identified during the simulation year occurred at high enough annual frequency $\left(>15\right.$ days $\mathrm{yr}^{-1}$ ) to be used for our analyses ["Cyprus Low to the North (Shallow)", "High to the North", "High to the West", "Persian Trough (Medium)", "Persian Trough (Weak)", "Red Sea Trough with the Central Axis", and "Red Sea Trough with the Eastern Axis"]. The Cyprus Low Troughs are part of the winter lows contributing winter rainfall of high intensity. The Persian Troughs are characterized by persistent summer weather conditions in the eastern Mediterranean, including Jordan and Iraq (Alpert et al. 1990). The Red Sea Troughs are situated over the Red Sea area throughout the year and deepen northward during winter (e.g., Dayan et al. 2001). Fifteen randomly sampled days from each class were used for the analysis.

Several statistical significance tests were applied to identify differences in model performance: the KruskalWallis $H$ test, a nonparametric method for testing whether samples originate from the same distribution (Breslow 1970), was used to compare forecast performance between lead days, seasons and synoptic pressure regimes; the Wilcoxon signed-rank test, a nonparametric method for examining the median difference in elements for two populations that are paired or dependent on one another (Siegal 1956), was used to compare WRF performance to WRF after statistical bias corrections (described in section 2e); and the Mann-Whitney $U$ test, a nonparametric method for examining the difference in medians for two independent populations (Mann and Whitney 1947), was used to compare WRF forecast performance to observation-based predictions (described in section 2f).

\section{e. $W R F_{B C}: A$ hybrid WRF-observation approach}

We hypothesized that systematic forecast errors in $W_{s}$, which can be attributed to local effects and do not change sharply with time, can be corrected using past observational and WRF forecast data. Based on the fact that results showed low temporal dependence of the $W_{s}$ FB (section 3a) we investigated the potential improvement in $\mathrm{ET}_{0}$ forecast by applying a bias correction to the simulated $W_{s}$ based on the relative model-observation $W_{s}$ bias prior to forecast. This bias correction was performed by applying the following steps. First, the mean relative bias $r$ of the 5 days prior to the forecast was calculated individually for each meteorological station, considering that the bias in $W_{s}$ was found to be highly affected by local conditions. The mean relative bias at each hour of the day $(r)$ was calculated as

$$
r=\sum_{i=\tau}^{n+\tau} \frac{P_{i}^{\text {model }}-P_{i}^{\text {obs }}}{n P_{i}^{\text {model }}},
$$


TABLE 2. Synoptic classes defined on a daily basis using sea level pressure data at 1200 UTC (after Alpert et al. 2004) and their annual frequency in 2013. Highlighted in bold are the most frequent synoptic classes, which are analyzed in Fig. 5.

\begin{tabular}{rrll}
\hline \hline No. & Annual frequency (2013) & \multicolumn{1}{c}{ Synoptic class } & Abbreviation \\
\hline 1 & 13 & Cyprus Low to the North (Deep) & CND \\
$\mathbf{2}$ & $\mathbf{1 8}$ & Cyprus Low to the North (Shallow) & CNS \\
3 & 4 & Cyprus Low to the South (Deep) & CSD \\
4 & 15 & High over Israel (Central) & HE \\
5 & 14 & High to the East & HN \\
$\mathbf{6}$ & $\mathbf{2 6}$ & High to the North & HW \\
$\mathbf{7}$ & $\mathbf{4 3}$ & High to the West & LED \\
8 & 8 & Low to the East (Deep) & LES \\
9 & 9 & Low to the East (Shallow) & PTD \\
10 & 5 & Persian Trough (Deep) & PTM \\
$\mathbf{1 1}$ & $\mathbf{6 3}$ & Persian Trough (Medium) & PTW \\
$\mathbf{1 2}$ & $\mathbf{6 5}$ & Persian Trough (Weak) & RSC \\
$\mathbf{1 3}$ & $\mathbf{2 8}$ & Red Sea Trough with the Central Axis & RSE \\
15 & $\mathbf{3 5}$ & Red Sea Trough with the Eastern Axis & RSW \\
16 & 4 & Red Sea Trough with the Western Axis & SC \\
17 & 3 & Sharav Low over Israel (Central) & SW \\
\hline
\end{tabular}

where $i$ is the hourly time index (equals 0 for the forecasted day and rises positively backward; e.g., if $i=0$ at 1300 UTC 26 March 2013, $i=1$ at 1300 UTC March $252013), \tau$ is the forecast lead day, and $n$ is the number of averaged preceding days; $n$ was set to 5 days as a compromise between increasing IOA and increasing $|\mathrm{FB}|$ with increasing $n$ ( $n$ between 2 and 14 days was tested; presented in Fig. A1). Second, $W_{s}$ was corrected based on an inversion of Eq. (6):

$$
P^{\text {corrected }}=(1+r) P^{\text {model }}
$$

where $P^{\text {corrected }}$ and $P^{\text {model }}$ are the corrected and model hourly $W_{s}$, respectively. Considering that recalculation of the simulated $\mathrm{ET}_{0}$ using the bias-corrected $W_{s}$ led to significant improvement of the $\mathrm{ET}_{0}$ forecast, we further tested the benefit of using this approach in forecasting $\mathrm{ET}_{0}$ at locations far from any station, by interpolating the corrected $\mathrm{ET}_{0}$ to every location using the inverse distance weighting (IDW) interpolation method (section $2 \mathrm{f}$ ). Third, the quality of the WRF forecast after correction and interpolation using this hybrid approach $\left(\mathrm{WRF}_{\mathrm{BC}}\right)$ was tested. Daily sums of $\mathrm{ET}_{0}$ obtained by $\mathrm{WRF}_{\mathrm{BC}}$ $\left(\mathrm{ET}_{0-\mathrm{BC}}\right)$ were compared with the corresponding values which were obtained by WRF, namely, without bias corrections.

\section{f. IDW interpolation observation-based forecast method}

Since many farmers irrigate based on recent ET in order to replenish what was evapotranspired from the agricultural field, rather than using weather forecasts, we also investigated the potential of using past observations to provide $\mathrm{ET}_{0}$ predictions for areas far from meteorological stations. The quality of such an approach was evaluated by using observations from meteorological stations in the following steps: (i) calculation of $\mathrm{ET}_{0}$ for each of the 29 meteorological stations; (ii) interpolation for each station was done by using the hourly $\mathrm{ET}_{0}$ of the other 28 stations and interpolating the data by use of the IDW interpolation method (Shepard 1968) given by

$$
z_{p}=\frac{\sum_{i=0}^{n}\left(\frac{z_{i}}{d_{i}^{p}}\right)}{\sum_{i=0}^{n} \frac{1}{d_{i}^{p}}}
$$

where $z_{p}$ is the interpolated value, $z_{i}$ is the examined value at station $i, n$ is the number of stations used for interpolation (28 in our case), $d_{i}$ is the distance of station, $i$ is the sequential station number, and $p$ is a power parameter, set to $p=2$ in this case (proposed as optimal by Shepard 1968); (iii) interpolation of data from previous days to "forecast" $\mathrm{ET}_{0}$ with the IDW method, that is, for $\tau$ lead-day forecast of $\mathrm{ET}_{0}$ at station $x$ at time $t$, the hourly $\mathrm{ET}_{0}$ was interpolated from all other stations except for station $x$ at time $=t-\tau$ days; and (iv) comparison of the interpolated daily $\mathrm{ET}_{0}$ (ET $\left.\mathrm{E}_{0-\mathrm{IDW}}\right)$ to the WRF daily $\mathrm{ET}_{0}$. Note that the IDW was also used for our hybrid approach (section 2e). The three $\mathrm{ET}_{0}$ prediction methods are summarized in Table 1. 


\section{Results and discussion}

The PM variables and $\mathrm{ET}_{0}$ forecasted by WRF were analyzed first on an hourly basis with respect to lead time, location, seasonality, and predominant synoptic regime to provide insight into the WRF Model's performance (section 3a). Then, the daily $\mathrm{ET}_{0}$, calculated as the daily sum of hourly values, was compared to daily $\mathrm{ET}_{0-\mathrm{IDW}}$ and $\mathrm{ET}_{0-\mathrm{BC}}$ in order to compare the WRF Model to the observation-based model and the hybrid bias-corrected WRF Model (IDW and $\mathrm{WRF}_{\mathrm{BC}}$, respectively, sections $2 \mathrm{f}$ and $2 \mathrm{e}$ ). This comparison provided a better understanding of the WRF Model as a tool for irrigation decision-making because daily $\mathrm{ET}_{0}$ values are usually used by farmers (section $3 b$ ).

\section{a. Evaluation of WRF performance compared to observations}

\section{1) TEMPORAL AND SPATIAL EVALUATION OF WRF MODEL}

Figure 2 presents a general comparison between the hourly WRF simulated and observed meteorological parameters that are required to calculate $\mathrm{ET}_{0}$ using FAO-56 PM [see Eq. (1)], as well as FAO-56 PM-ET itself, for the year 2013. Note that in contrast to the other analyses which are described in successive figures and present $W_{s}$, Fig. 2 presents the zonal and meridional wind vector components at $2 \mathrm{~m}$ to gain insight into the potential causes of $W_{s}$ forecast errors which are related to $W_{s}$ components ( $U$ and $V$, respectively; Figs. $2 \mathrm{~d}$,e).

The agreement between the model simulations and observations for all parameters (Fig. 2) deteriorated with lead day, but not significantly for all of the parameters ( $p$ value $>0.05$ ). The $R_{s}, T$, and $\mathrm{RH}$ forecasts were not robust to lead days. The WRF $\mathrm{ET}_{0}$ forecast showed a high IOA with respect to $\mathrm{ET}_{0}$ evaluated based on the observations (mean and median values higher than 0.97) and remained almost unchanged with no significant deterioration over the 4-day forecast period (Kruskal-Wallis $p$ value $=0.13$, with a null hypothesis that stations IOAs for every lead day emerge from identical populations). Robustness of the forecast to lead time is of key importance when relying on forecasts for irrigation scheduling, because irrigation intervals for most agricultural crops are longer than 1 day. IOA values for $T$ were similar to those of $\mathrm{ET}_{0}$. This is to be expected because $T$ is usually the most dominant parameter in the PM equation (Piper 1989). Both $U$ and $V$ were robust to lead days (Figs. 2d,e). The zonal wind $U$ had lower IOA values and larger variation than the meridional wind $V$ with some stations having IOA $<0.7$. Mean $W_{s}$ IOA values were $<0.75$ for all lead days (not presented), lower than each of its components, $U$ and $V$. Parameter $W_{s}$ is used for the FAO-56 $\mathrm{ET}_{0}$ calculation, but because the WRF Model state variables are $U$ and $V$, it is important to be aware of their forecast accuracy and try to improve it by optimizing model parameterization. IOA values for $R_{s}$ were high but deteriorated with lead days, as the $T$ and RH IOA values. Since $R_{S}$ affects $T$ and in turn RH, improving $R_{s}$ forecast accuracy can lead to improvement of $T$ and $\mathrm{RH}$ forecasts as well.

The accuracy of the WRF output parameters varied with lead time but also spatially. Figure 3 presents the IOA between the simulated 2-lead-day forecast of all meteorological parameters required for $\mathrm{ET}_{0}$ calculation and observations, as well as for the calculated $\mathrm{ET}_{0}$ and precipitation, individually for each meteorological station. There was an increase in the WRF IOA of $R_{s}$ from north to south (Fig. 3a). When moving southeast from the coastline, the climate changes from Mediterranean to arid, and it is therefore reasonable to hypothesize that errors in cloud cover prediction affected the $R_{s}$ prediction to a lesser degree in those arid areas. IOA of the $T$ forecast was almost unchanged with location but slightly lower along the coastline (Fig. 3b), potentially for the same reason that the $R_{s}$ IOA was lower in the coastal area. The $\mathrm{RH}$ forecast along the coastline was poorer as well (Fig. 3c), with mean IOA of 0.814 . Since mean $q$ IOA for the coastal locations was 0.92 (not presented in the figures) low RH can be attributed to lower $T$ IOA. IOA values for $W_{s}$ were generally low and varied across a large range (Fig. 3d; $0.45<$ IOA $<$ 0.86 ), with no visible latitude-longitude trend. They were higher along the southern coast and lower for the northern Galilee mountainous area (stations 12, 15, 25) and the southeast Arava Valley (stations 24, 28) (Fig. 3d). The complex terrain features of the Galilee and Arava areas may have played a role in the poorer $W_{s}$ predictions. Whereas we could not identify lower $W_{s}$ forecasting skill for stations located in a more complex terrain area relative to others, overprediction of $W_{s}$ by WRF is extensively documented and can arise from unresolved short-scale turbulent flow, which can be further induced by topographic features and surface roughness (see section 1). Hourly $\mathrm{ET}_{0}$ IOA values were high $(>0.95)$ and without any distinguishable geographic gradient (Fig. 3e). The two southeastern stations in the Arava Desert scored lowest for $\mathrm{ET}_{0}$ IOA (stations 24, 28) even though these locations did not score the lowest IOA for all or most other PM parameters which were used to calculate $\mathrm{ET}_{0}$. This indicates inconsistency between $\mathrm{PM}-\mathrm{ET}_{0}$ and $\mathrm{PM}$ parameters, which is expected when one considers that errors in these meteorological parameters can cancel each other out (e.g., positive $T$ bias and negative $W_{s}$ bias may result in an unbiased $\mathrm{ET}_{0}$ ). Therefore, each PM parameter should be predicted accurately to provide a reliable $\mathrm{ET}_{0}$ forecast. 

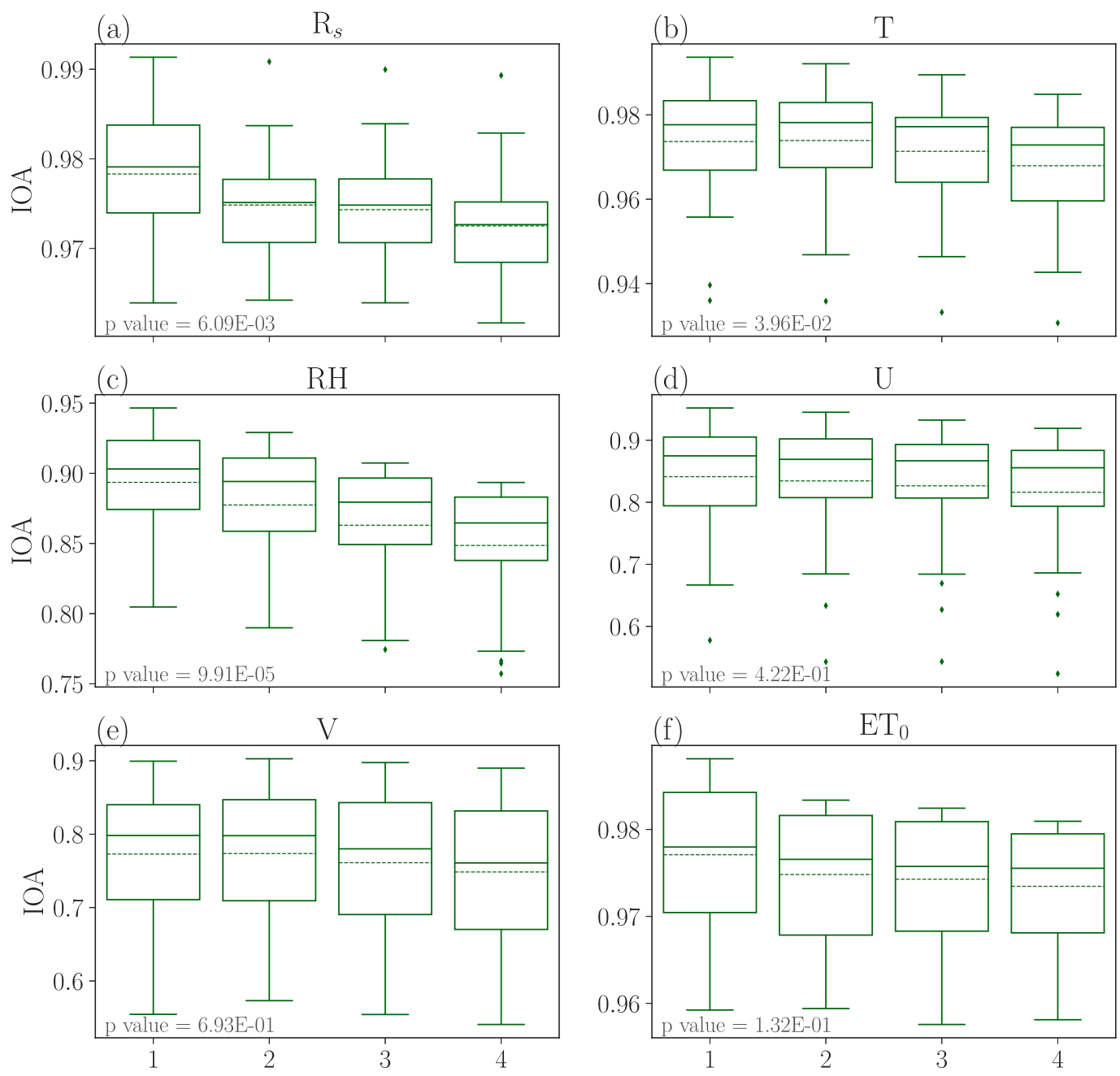

Lead day

FIG. 2. Boxplots of WRF Model vs observation IOA for hourly (a) solar radiation $R_{s}$, (b) 2-m temperature $T$, (c) relative humidity $(\mathrm{RH})$, (d) zonal $U$ and (e) meridional $V$ 2-m wind components, and (f) potential evapotranspiration $\left(\mathrm{ET}_{0}\right)$. Results are compared for each of 1-4-lead-day forecasts. Boxplots indicate the median and 25 th and 75 th percentiles. Whiskers extend to 1.5 times the interquartile range. Dashed lines mark the mean values. The $p$ values of the Kruskal-Wallis $H$ test comparing lead days are presented for each parameter.

Figure $3 \mathrm{f}$ presents AUC values calculated for detection of daily precipitation $>5 \mathrm{~mm} \mathrm{day}^{-1}$ for each station. Some stations are absent from the figure since no days with rain above threshold occurred in 2013 at those locations. AUC score at station 24 was lowest but with only 2 days above-threshold rain. All other stations scored AUC higher than 0.89 , with 0.95 on average. With AUC $=0.5$ meaning zero ability for prediction, this can be considered a high score for agricultural irrigation and fertigation decision-making, with the benefit further depending on crop, soil, time of year, and economic considerations. Note that in addition to water conservation, the rain-event forecast is important for suppression of fertigation prior to rain events to prevent fertilizer washout under the crop root zone. AUC for rain-event predictions for other than 2 lead days was also calculated (not presented in this paper) and comparison of 1-4 lead days did not show significant differences in AUC values, which ranged roughly between 0.95 and 0.97 (Kruskal-Wallis $p$ value $=0.15$ ).

\section{2) SEASONAL AND SYNOPTIC EFFECTS ON

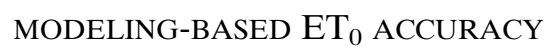

In this section we explore the impact of season and different synoptic pressure regimes on the WRF prediction of PM parameters and $\mathrm{ET}_{0}$ evaluation. Characterizing 

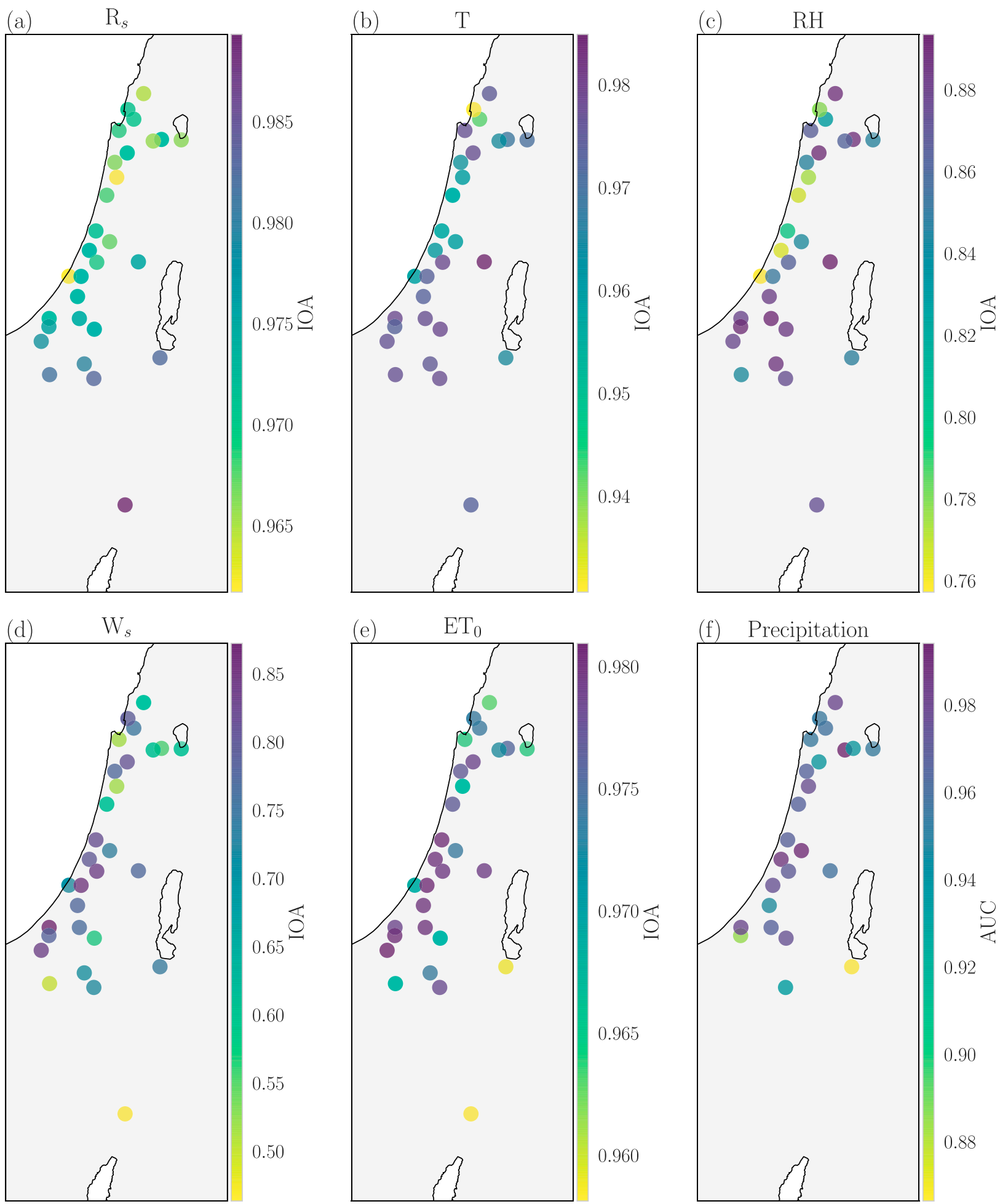

FIG. 3. IOA for hourly (a) solar radiation $R_{s}$, (b) 2-m air temperature $T$, (c) relative humidity (RH), (d) wind speed $W_{s}$, and (e) potential evapotranspiration $\left(\mathrm{ET}_{0}\right)$ between observed and 2-lead-day predictions, and (f) AUC for 2-lead-day precipitation predictions. For (a)-(e) each marker represents the annual IOA between meteorological station and nearest grid point of WRF output; for (f) each dot represents the AUC of the ROC curve for daily precipitation $>5 \mathrm{~mm}$ at the nearest grid point of each meteorological station. Two stations in the southern desert did not have a single daily measurement of $>5$-mm rain and are therefore not presented on the map. Note that the colorbar scale is different for each panel. 
the effects of season and synoptic pressure systems on WRF results can be important in identifying the model's limitations, thereby enabling its improvement. Figure 4 presents boxplots for IOA and FB obtained by comparing 2-lead-day WRF Model forecasts and observations for all stations classified according to season of the year. Figure $4 \mathrm{a}$ indicates that the means (and medians) of IOA scores for $\mathrm{ET}_{0}$ were highest on summer days and of $\mathrm{FB}$, closer to 0 (Fig. 4b), but with large variability between locations, as indicated by the large whisker spans, most scoring between -0.5 and $0.75 \mathrm{FB}$. Winter days' $\mathrm{ET}_{0}$ forecast had the lowest IOA scores and highest FB (Figs. 4a,b). Due to large FB and IOA variability across locations, it is hard to conclude that WRF performed better during the summer period only due to arbitrary compensation of IOA and FB across different stations; to draw this conclusion, the seasonal variation in performance should be examined for each station individually on a multiyear basis. Moreover, it should be emphasized that some stations do have a large $\mathrm{ET}_{0} \mathrm{FB}$ in summer but considering that this term represents the normalized $\mathrm{ET}_{0}$ bias, the actual bias value can be substantially higher than in other seasons. This is of particular importance considering that in summer, most crops depend heavily on both temporally and quantitatively precise irrigation. It should be further emphasized that the low bias of $\mathrm{ET}_{0}$ (mean $\mathrm{FB} \approx 0$ ) and relatively high IOA are not due to better model performance for the PM meteorological parameters but rather to compensation between errors in the individual parameters. This explanation is supported by negative $R_{s}$ and $T \mathrm{FB}$ values but positive $\mathrm{RH}$ and $W_{s} \mathrm{FB}$ values for the summer, resulting in seemingly better model performance.

In the following, the seasonal differences in the impact of simulated PM parameters on WRF $\mathrm{ET}_{0}$ forecast is analyzed. The $T$ IOA was lower and more varied among stations in the summer and winter seasons compared to spring and autumn (Fig. 4a). Mean and median FB of the $T$ forecast was positive in autumn and winter and negative in spring and summer, with very high variability on winter days compared to other seasons (Fig. 4b). The seasonal trend of hourly $\mathrm{ET}_{0} \mathrm{FB}$ was similar to that of $T$ FB but higher and mostly positive. The RH FB trend between seasons was anticorrelated to those of $T$ and $\mathrm{ET}_{0} \mathrm{FB}$ (as well as to the $q \mathrm{FB}$ trend, not presented). $\mathrm{RH}$ bias was enhanced by the $T$ bias, which contributes to the RH bias in the opposite direction (i.e., warm bias will contribute to negative RH bias). The $W_{s}$ forecast for all seasons was poor in terms of both IOA and FB, and without significant differences among seasons (Fig. 4). This is in contrast to all other investigated PM parameters and supports the assumption that a bias in $W_{s}$ is dominated primarily by local terrain or land-use data not properly taken into account by the WRF Model, regardless of seasonal dependence. The high positive $W_{s}$ bias seems to be a major contributor to the $\mathrm{ET}_{0}$ bias because the FB of all other PM parameters was mostly negative or around zero across seasons, and yet $\mathrm{ET}_{0} \mathrm{FB}$ was higher than that of all PM parameters except of $W_{s}$.

The mean and median IOA scores for $R_{s}$ were lower in the winter than in the other seasons, and its FB was negative for all seasons and less negative in autumn and spring (Figs. 4a,b). Considering the higher cloud cover in the studied area in winter compared to summer, the lowest $R_{s}$ IOA (and highest absolute FB) during the winter and highest during the summer further supports our previous assumption that errors in predicting the cloud fraction are a major contributor to the errors in $R_{s}$ forecast, as also supported by previous studies (e.g., Lara-Fanego et al. 2012; Kosmopoulos et al. 2015).

Our analysis further indicated a significant impact of synoptic conditions on the different meteorological PM parameters. Figures $5 \mathrm{a}$ and $5 \mathrm{~b}$ investigate the IOA and FB, respectively, with respect to observations for the seven most frequent synoptic classes in 2013 (see Table 2). Figure 5 shows statistically significant differences under the different presented synoptic scenarios, for all PM variables and $\mathrm{ET}_{0}$ IOA (Fig. 5a), whereas in terms of FB, only $W_{s}$ did not show statistically significant differences under the different synoptic scenarios (Fig. 5b). On Cyprus Low to the North (Shallow) days, IOA values for forecasted $R_{s}$ were low and varied greatly, but the FB remained low and close to zero. On Red Sea Trough (with Central and Eastern axis) days, the WRF predicted $R_{s}$ had the lowest IOA and highest $|\mathrm{FB}|$ whereas on Persian Trough (Weak and Medium) days, the highest IOA and lowest $|\mathrm{FB}|$ were achieved for this parameter. These differences in $R_{s}$ forecast performance between the Persian and Red Sea Trough days can be attributed to higher aerosol optical thickness which affects radiation transfer in Red Sea pressure system periods compared to those of the Persian systems, as described in Carmona and Alpert (2009). In agreement with the seasonal analysis, $T$ and $\mathrm{RH}$ forecasts had opposing FB trends due to $q$ having an opposing FB trend to $T$, reinforced by the biased $T$ component of $\mathrm{RH}$. This emphasizes the notion of a strong impact of the $T$ forecast on the $\mathrm{ET}_{0}$ forecast, due to the fact that RH and $T$ are intertwined.

$\mathrm{ET}_{0}$ had lower IOA and higher FB values for Red Sea Troughs versus Persian Trough periods. The IOA trend for $\mathrm{ET}_{0}$ with synoptic classes was comparable with that for $R_{s}$ and $W_{s}$ forecast, whereas FB of the other parameters had relatively low (absolute) values. In agreement with previous studies (e.g., Berkovic 2016) the Red Sea Trough days were drier than the Persian Trough days 

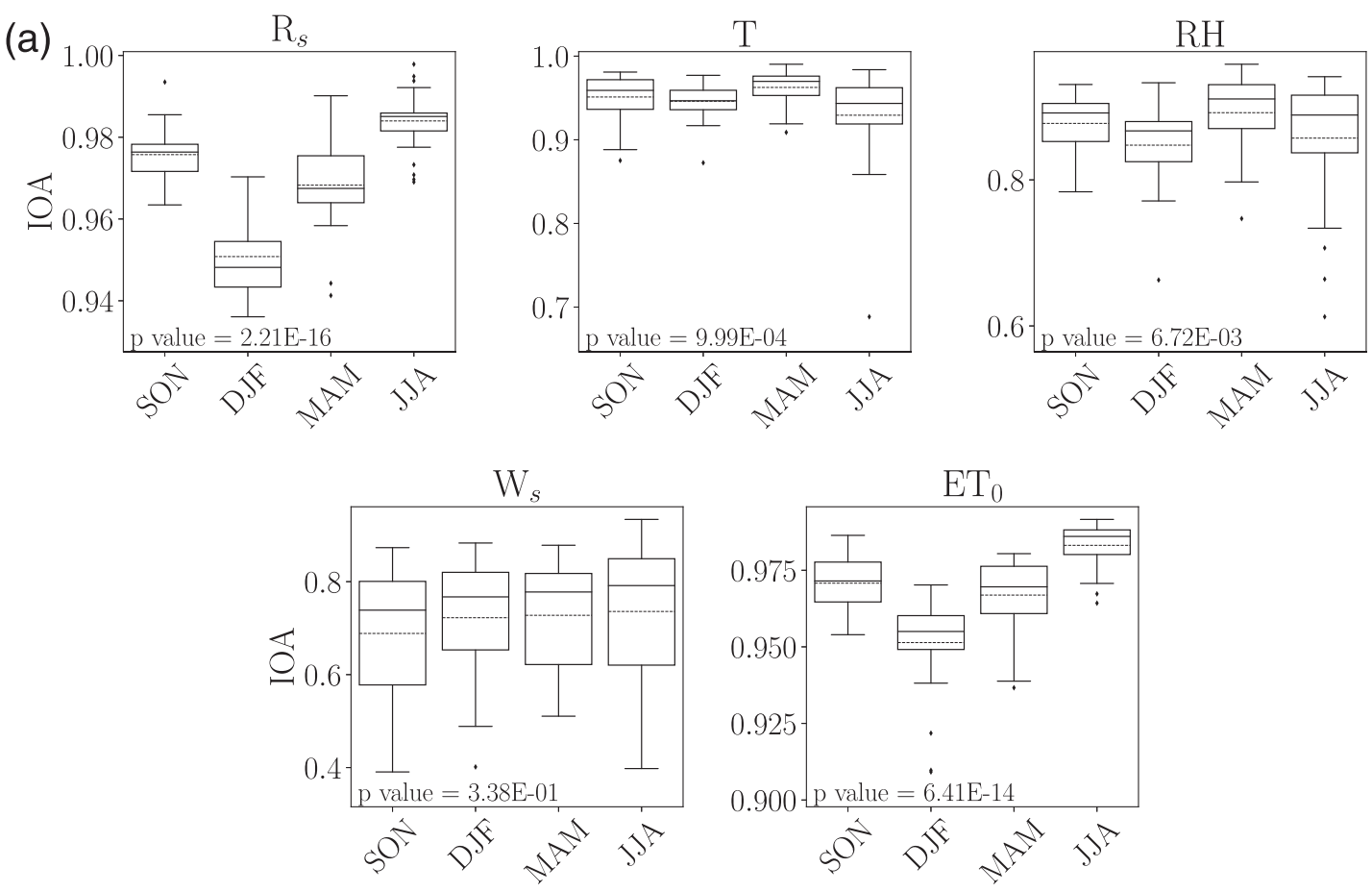

Season
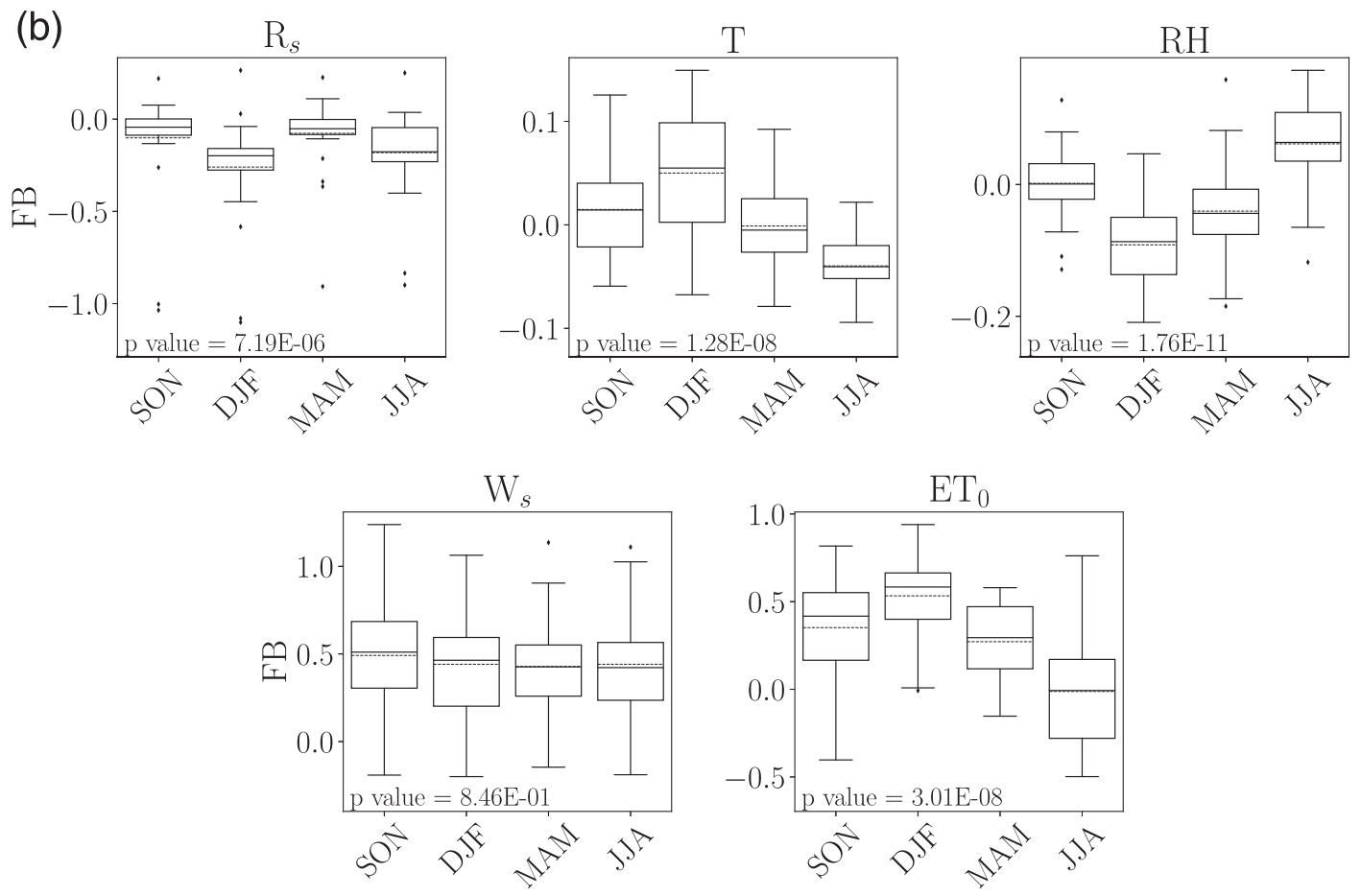

Season

FIG. 4. Boxplots for (a) IOA and (b) FB at station locations, by season, for hourly solar radiation $R_{s}, 2$-m temperature $T$, air relative humidity $(\mathrm{RH})$, wind speed $W_{s}$, and potential evapotranspiration $\left(\mathrm{ET}_{0}\right)$ of the 2-lead-day forecast. Boxplots indicate the median and 25th and 75 th percentiles. Whiskers extend to 1.5 times the interquartile range. Dashed lines mark the mean values. The $p$ values of the Kruskal-Wallis $H$ test comparing seasons are presented for each parameter. Autumn is September-November (SON), winter is December-February (DJF), spring is March-May (MAM), and summer is June-August (JJA). 
(a)

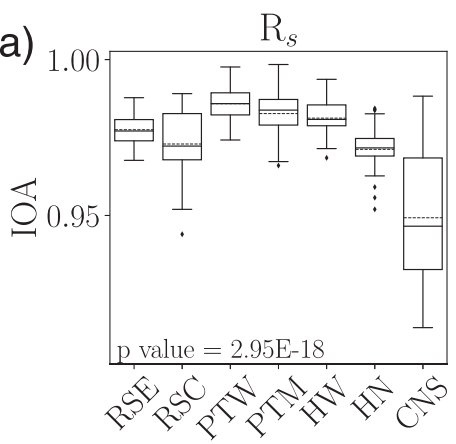

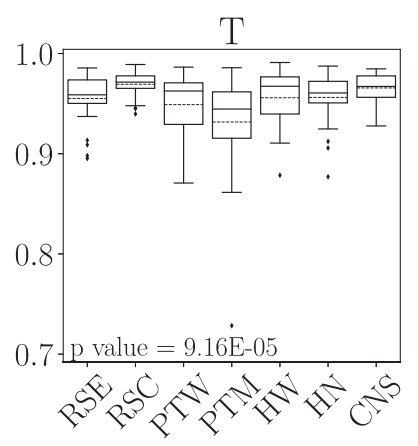

$\mathrm{RH}$

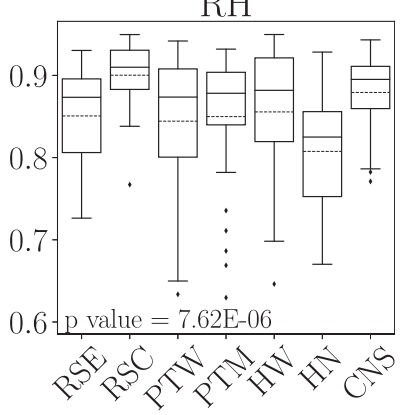

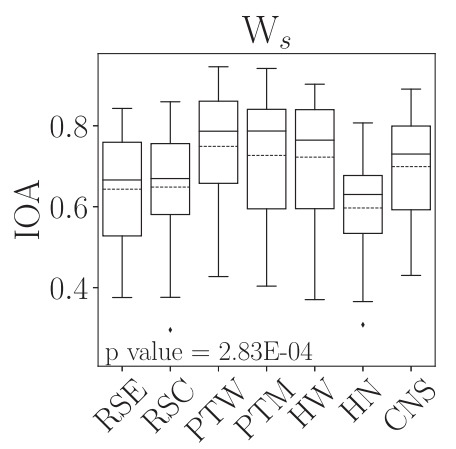

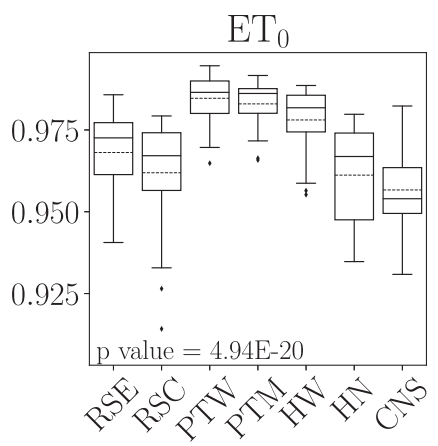

Synoptic class
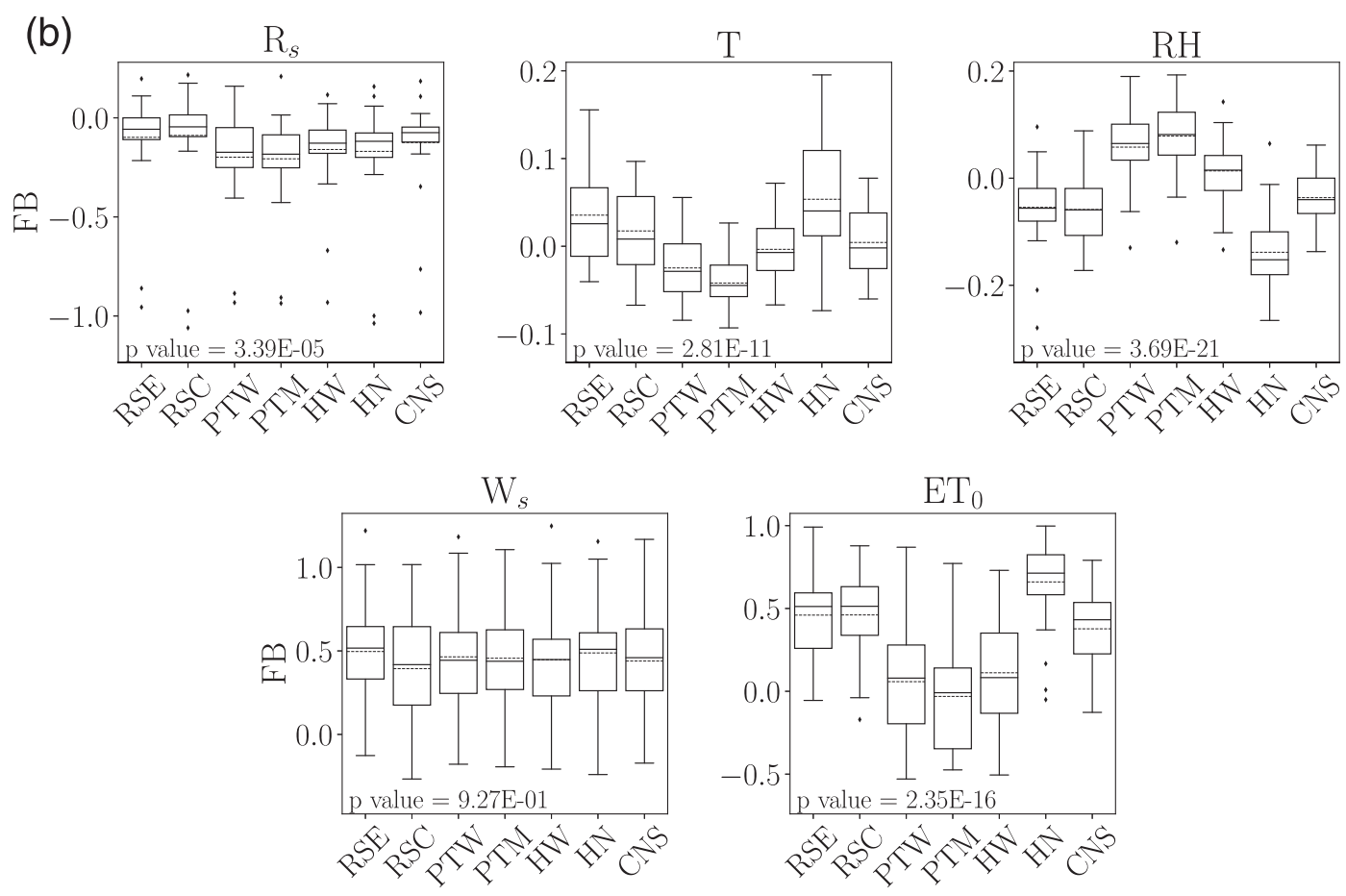

Synoptic class

FIG. 5. Boxplots for (a) IOA and (b) FB at all stations for each of seven synoptic pressure system classes examined (detailed in Table 2) performed for hourly solar radiation $R_{s}, 2-\mathrm{m}$ temperature $T$, air relative humidity (RH), wind speed $W_{s}$, and potential evapotranspiration $\left(\mathrm{ET}_{0}\right)$ of the 2-lead-day WRF forecast. Boxplots indicate the median and 25 th and 75 th percentiles. Whiskers extend to 1.5 times the interquartile range. Dashed lines mark the mean values. The $p$ values of the Kruskal-Wallis $H$ test comparing synoptic classes are presented on each graph. See Table 2 for synoptic class abbreviations. 


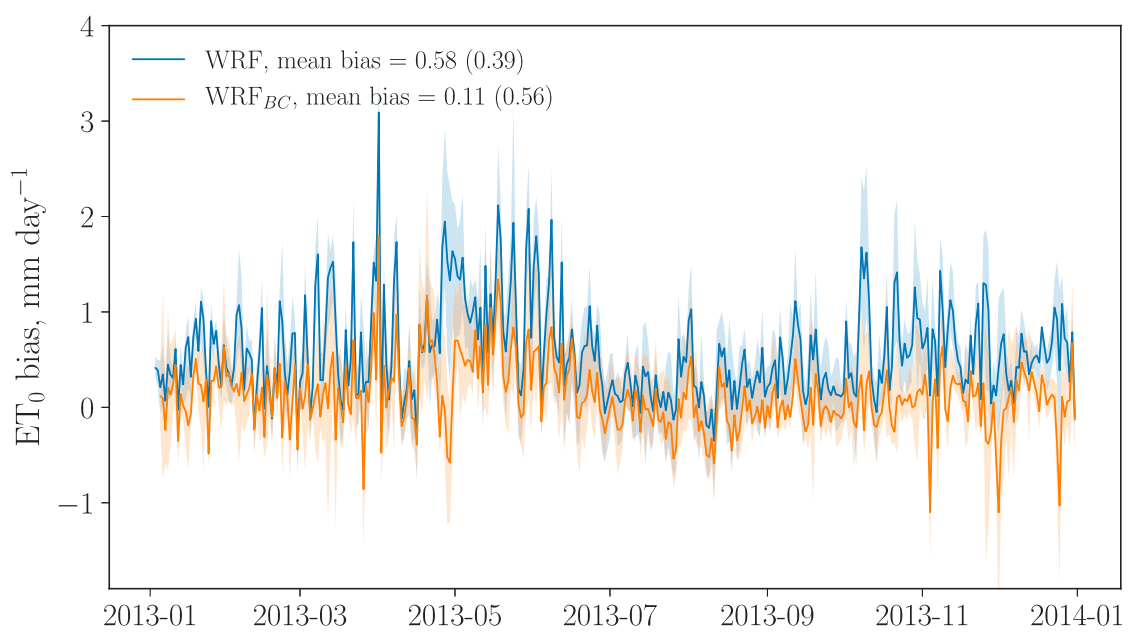

FIG. 6. Daily $\mathrm{ET}_{0}$ bias values throughout 2013 between WRF and observations (WRF) and the applied hybrid approach $\left(\mathrm{WRF}_{\mathrm{BC}}\right.$; section $\left.2 \mathrm{e}\right)$ and observations of the 2-lead-day forecast. Curves are daily mean values for all weather stations (thick lines) together with the 0.25 and 0.75 confidence levels. Given are the annual mean (thick curve) and standard deviation in parentheses.

(56\% versus $63 \%$ observed mean $\mathrm{RH}$ across stations and $51 \%$ versus $67 \%$ WRF mean RH across stations, respectively). Thus, positively biased $W_{s}$ predictions had a stronger effect on $\mathrm{ET}_{0}$ for Red Sea Trough days as reflected by Eq. (1).

\section{b. Daily potential evapotranspiration}

Daily $\mathrm{ET}_{0}$ was computed by summing positive hourly $\mathrm{ET}_{0}$ values. We tested the potential improvement of the $\mathrm{ET}_{0}$ forecast by applying the bias correction scheme depicted in section 2e. Figure 6 compares WRF daily $\mathrm{ET}_{0}$ with corresponding observed values, with and without applying the hybrid approach, namely $\mathrm{WRF}_{\mathrm{BC}}$ versus WRF. WRF seemed to severely overpredict daily $\mathrm{ET}_{0}\left(0.58 \mathrm{~mm} \mathrm{day}^{-1}\right.$ annual mean $)$ compared to $\mathrm{WRF}_{\mathrm{BC}}$. WRF daily $\mathrm{ET}_{0}$ biases were mostly positive throughout the year for all stations-higher in spring and summer with peaks of up to $3 \mathrm{~mm} \mathrm{day}^{-1}$. High biases of around $2 \mathrm{~mm} \mathrm{day}^{-1}$ were observed in October as well. $\mathrm{WRF}_{\mathrm{BC}}$ daily $\mathrm{ET}_{0}$ bias was lower $\left(0.11 \mathrm{~mm} \mathrm{day}^{-1}\right.$ annual mean), frequently underestimating $\mathrm{ET}_{0}$. Considering that mean daily $\mathrm{ET}_{0}$ was $4.2 \mathrm{~mm} \mathrm{day}^{-1}$ for the year 2013, WRF daily $\mathrm{ET}_{0}$ had about $13 \%$ annual mean bias when averaged over all locations, whereas this bias was reduced to about $3 \%$ with the $\mathrm{WRF}_{\mathrm{BC}}$. Improvement of $\mathrm{WRF}_{\mathrm{BC}} \mathrm{ET}_{0}$ forecast over that with WRF was not equal for all stations, as reflected by the increase in standard deviation of the $\mathrm{WRF}_{\mathrm{BC}}$ daily $\mathrm{ET}_{0}$ bias with respect to the WRF daily $\mathrm{ET}_{0}$ bias ( 0.56 versus $\left.0.39 \mathrm{~mm} \mathrm{day}^{-1}\right)$. Despite higher bias variability, higher than $1 \mathrm{~mm} \mathrm{~h}^{-1}$ |bias| of $\mathrm{WRF}_{\mathrm{BC}}$ daily $\mathrm{ET}_{0}$ was rare and it never exceeded $2 \mathrm{~mm}^{-1 a y}{ }^{-1}$ |bias|. During the hot and dry summer months of May-October (mean daily $\mathrm{ET}_{0}$ of $5.2 \mathrm{~mm} \mathrm{day}^{-1}$ across stations), underprediction of daily $\mathrm{ET}_{0}$ by $\mathrm{WRF}_{\mathrm{BC}}$ did not exceed $1 \mathrm{~mm}$ (overall mean bias of $0.13 \mathrm{~mm} \mathrm{day}^{-1}$ across stations for this period), and there is therefore no risk of underirrigation or crop water stress during the hot and dry summer period. Annual mean IOA score for the $\mathrm{WRF}_{\mathrm{BC}}$ daily $\mathrm{ET}_{0}$ was slightly improved from 0.92 (WRF) to $0.93\left(\mathrm{WRF}_{\mathrm{BC}}\right)$ (not presented here).

Figure 7 compares IOA for the daily $\mathrm{ET}_{0}$ forecast between WRF, the IDW interpolated forecast, which was based solely on observations (see section $2 \mathrm{f}$ ), and $\mathrm{WRF}_{\mathrm{BC}}$. The comparison was performed with respect to lead days and seasons. For all seasons and lead days (except the autumn 1-lead-day forecast; Fig. 7a), WRF achieved a significantly higher IOA than the IDW method (Fig. 7). IOA of the latter deteriorated with lead day, whereas WRF and $\mathrm{WRF}_{\mathrm{BC}} \mathrm{IOA}_{\mathrm{O}}$ values were almost unchanged with lead day, thus showing superiority in that respect. $\mathrm{WRF}_{\mathrm{BC}}$ showed significantly higher IOA values in the autumn months compared to WRF (for 4 lead days, Fig. 7a), but no significant differences for other seasons or number of lead days. While hourly WRF IOA values for $\mathrm{ET}_{0}$ were highest in the summer (Fig. 4a), daily IOA of $\mathrm{ET}_{0}$ were very low in the summer (Fig. 7d). This can be explained by the poorer performance of the WRF during the day versus nighttime, including dusk and dawn, when $\mathrm{ET}_{0}$ is $0 \mathrm{~mm} \mathrm{~h}^{-1}$ or very low and contributes little to the daily $\mathrm{ET}_{0}$. 

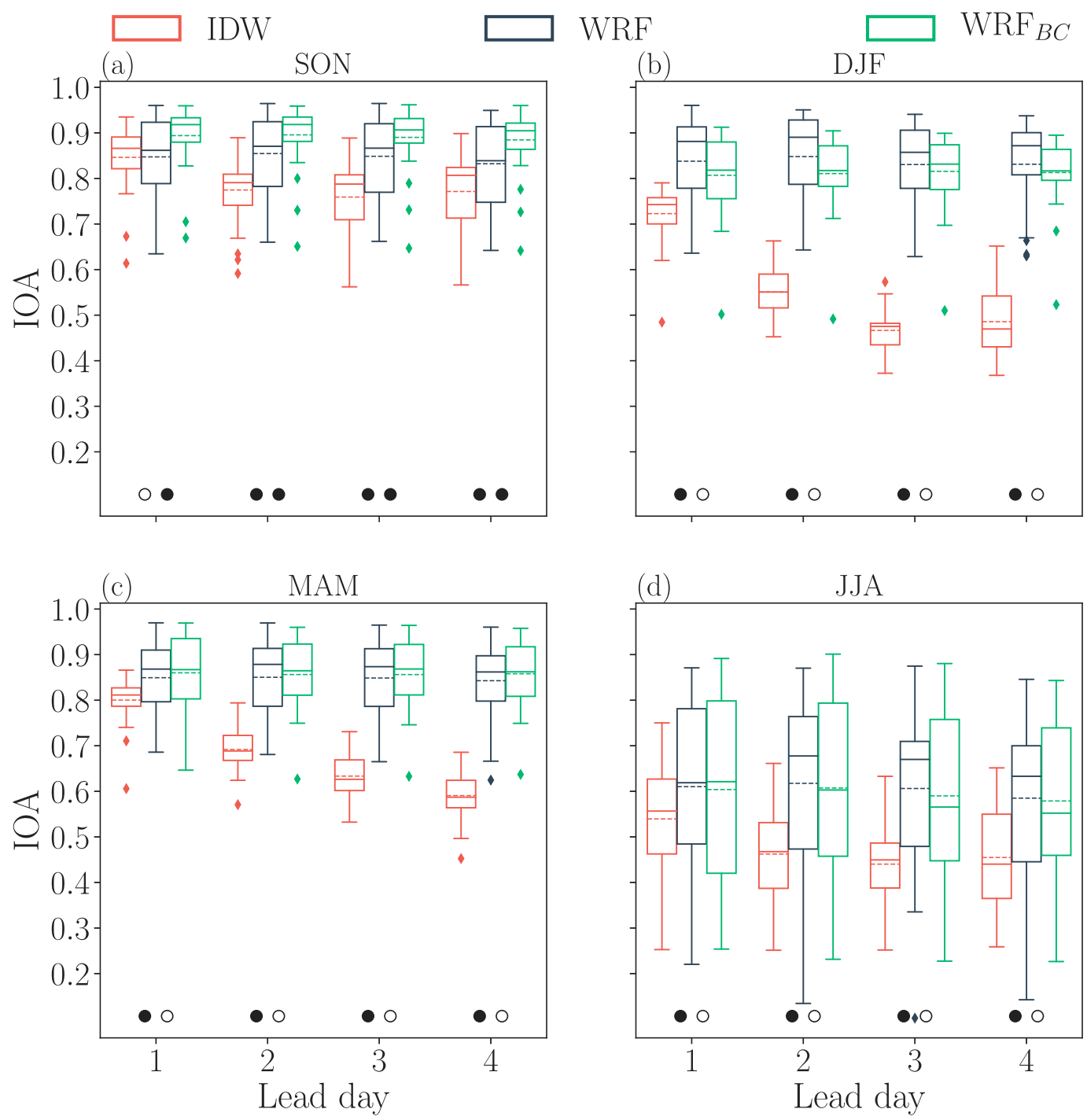

FIG. 7. Comparison of WRF Model to the IDW interpolation model (see section 2f) and to the bias-corrected WRF Model ( $\mathrm{WRF}_{\mathrm{BC}}$; see section 2e) in terms of model-observation IOA for daily $\mathrm{ET}_{0}$ with respect to season and lead day. Differences between WRF and IDW models were tested for significance with the Mann-Whitney $U$ test, and differences between $\mathrm{WRF}$ and $\mathrm{WRF}_{\mathrm{BC}}$ are tested with the Wilcoxon signed-rank test. Significant differences $(p$ value $<0.05)$ are marked with a filled circle between WRF and the alternative model and with an open circle for insignificant differences: (a) autumn (SON), (b) winter (DJF), (c) spring (MAM), and (d) summer (JJA).

Figure 8 compares the bias of the daily $\mathrm{ET}_{0}$ forecasted by WRF, IDW, and $\mathrm{WRF}_{\mathrm{BC}}$. Daily $\mathrm{ET}_{0}$ bias for the IDW method was negative on average for winter and spring, and positive for summer and autumn but low overall in absolute values. The WRF daily $\mathrm{ET}_{0}$ forecast exhibited a high positive bias for all seasons and cases. $\mathrm{WRF}_{\mathrm{BC}}$ predicted daily $\mathrm{ET}_{0}$ with a statistically significant lower bias for autumn, winter and spring days compared to WRF (Figs. 8a-c). For the summer months, the $\mathrm{WRF}_{\mathrm{BC}}$ bias was lower as well, although not statistically significantly so compared to the WRF forecast. At some of the peripheral locations (specifically stations 23 ,
27, 24, and 12), the absolute bias was higher for all seasons and lead days when predicted by the two methods involving interpolation (IDW method and $\mathrm{WRF}_{\mathrm{BC}}$ ). These stations also have large altitude differences compared to their surrounding stations (on the order of 100-200 m; positive for stations 23,12 and negative for stations 24,27 ). Thus, we can expect the $\mathrm{WRF}_{\mathrm{BC}}$ predictions to be less reliable at the edges of the station-network area. Overall, Fig. 8 indicates that IDW led to the lowest bias in forecasted $\mathrm{ET}_{0}$, but this resulted to a large extent from mutual cancellation of positive and negative biases in the $\mathrm{ET}_{0}$ forecast. Therefore, as a complementary approach to the 
$\square$ IDW
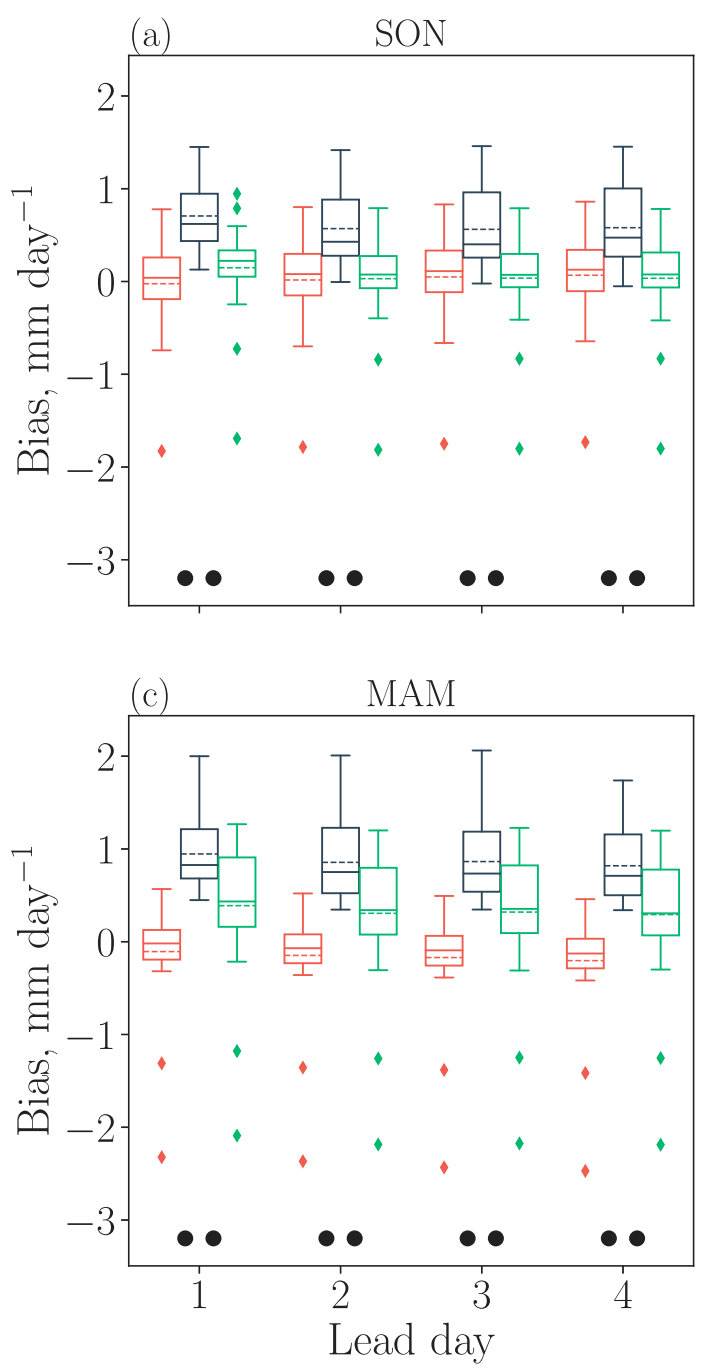

WRF

$\mathrm{WRF}_{B C}$
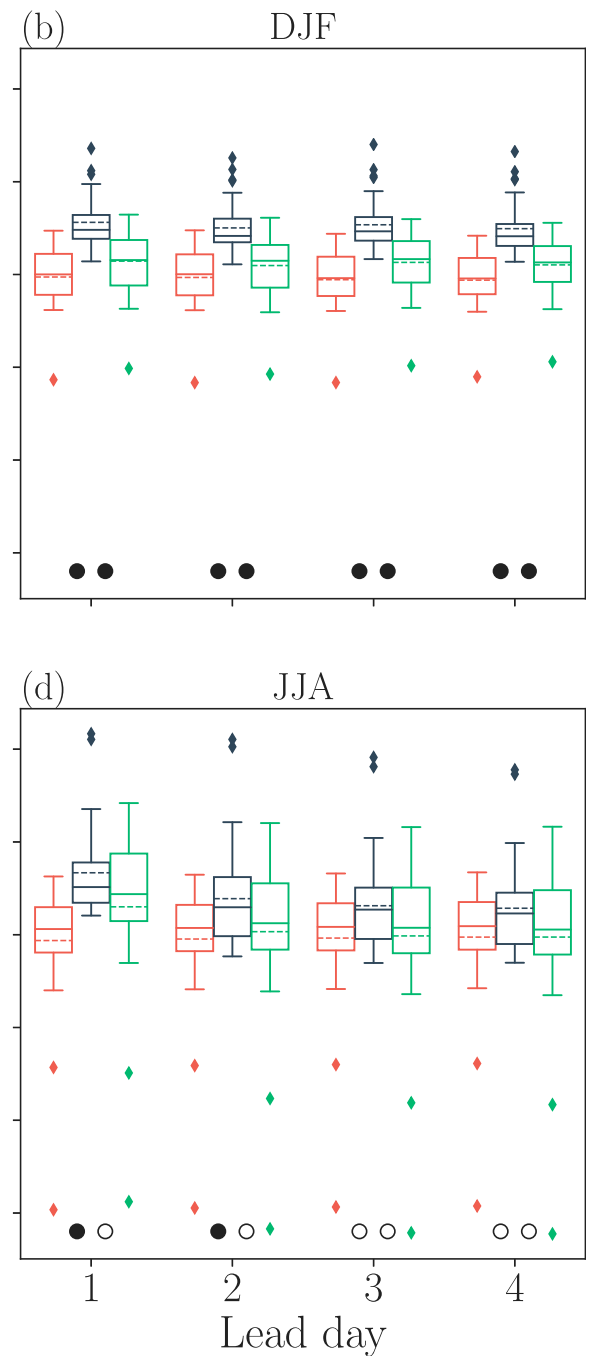

FIG. 8. Comparison of WRF Model to the IDW interpolation model (see section 2f) and to the bias-corrected WRF Model ( $\mathrm{WRF}_{\mathrm{BC}}$; see section 2e) in terms of model-observation bias for daily $\mathrm{ET}_{0}$ with respect to season and lead day. Differences between WRF and IDW models were tested for significance with the Mann-Whitney $U$ test, and differences between $W R F$ and $W_{R F}$ are tested with the Wilcoxon signed-rank test. Significant differences ( $p$ value $<0.05$ ) are marked with a filled circle between WRF and the alternative model and with an open circle for insignificant differences: (a) Autumn (SON), (b) winter (DJF), (c) spring (MAM), and (d) summer (JJA).

bias, we also evaluated the performance of the three models based on daily $\mathrm{ET}_{0}$ RMSE.

Figure 9 compares the RMSE of daily $\mathrm{ET}_{0}$ values forecasted by WRF, IDW, and $\mathrm{WRF}_{\mathrm{BC}}$. Daily $\mathrm{ET}_{0}$ RMSE for IDW had a higher mean and medians than that for $\mathrm{WRF}_{\mathrm{BC}}$, with growing differences with lead days for all seasons but summer. Daily $\mathrm{ET}_{0}$ RMSE for IDW was either significantly higher than that for WRF (Fig. 9b, and 2-4 lead days in Fig. 9c) or did not differ significantly from WRF, whereas daily $\mathrm{ET}_{0} \mathrm{RMSE}$ for $\mathrm{WRF}_{\mathrm{BC}}$ was either lower (autumn and spring; Figs. 9a,c) or did not differ significantly from that for WRF, thus making $\mathrm{WRF}_{\mathrm{BC}}$ the preferred model over IDW in all seasons, except summer in terms of RMSE. Because the study area has stable hot and dry weather throughout the summer, and the small differences across the different models with a generally lower bias during the summer months there is no benefit to any of the tested models in terms of RMSE in the summer, compared to other seasons.

The above demonstrates that when assessing a mix of positive and negative errors, both biases and RMSE 

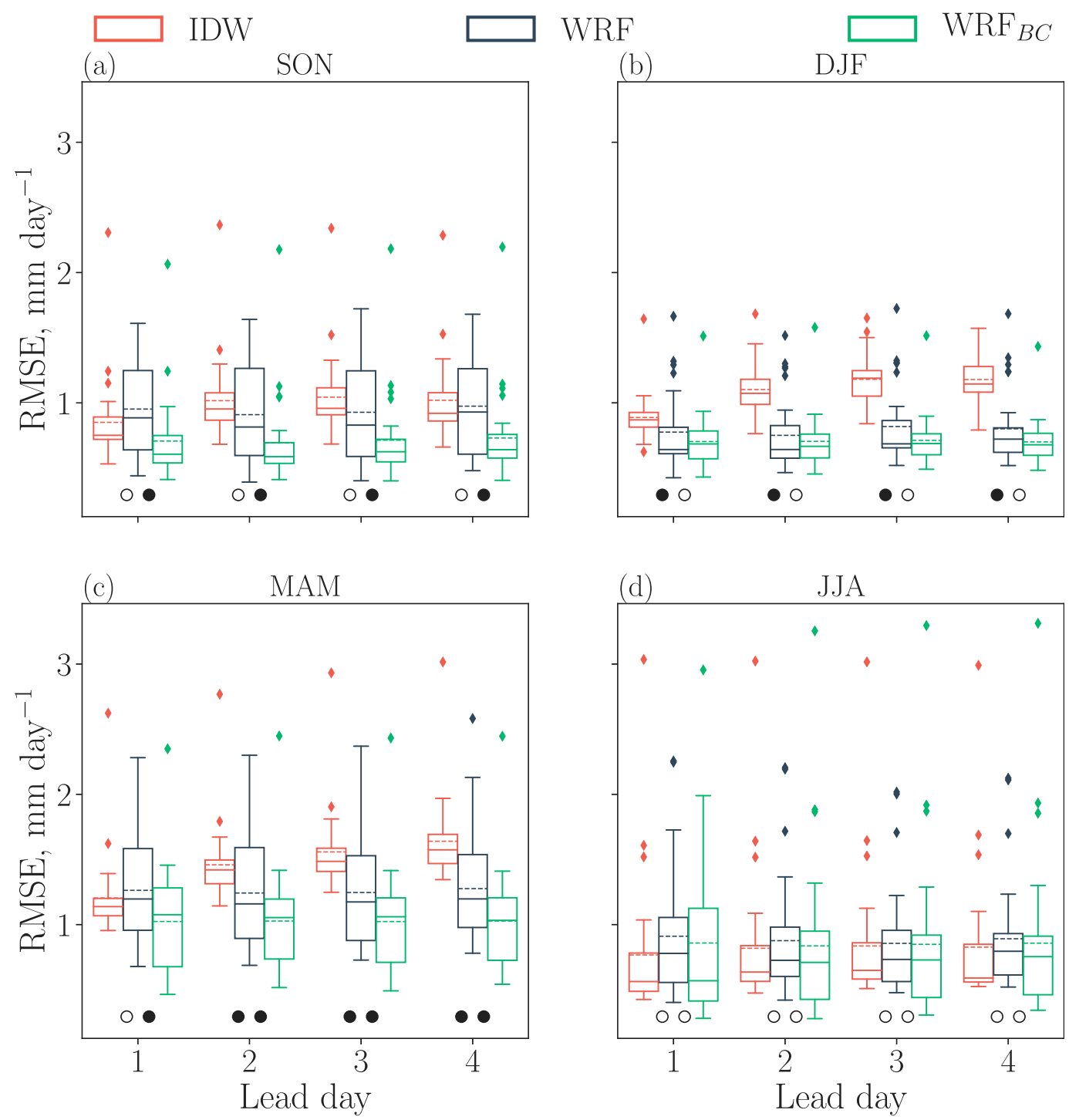

FIG. 9. Comparison of WRF Model to the IDW interpolation model (see section $2 \mathrm{f}$ ) and to the bias-corrected WRF Model ( $\mathrm{WRF}_{\mathrm{BC}}$; see section 2e) in terms of model-observation RMSE for daily $\mathrm{ET}_{0}$ with respect to season and lead day. Differences between WRF and IDW models were tested for significance with the Mann-Whitney $U$ test, and differences between $\mathrm{WRF}$ and $\mathrm{WRF}_{\mathrm{BC}}$ are tested with the Wilcoxon signed-rank test. Significant differences $(p$ value $<0.05)$ are marked with a filled circle between WRF and the alternative model and with an open circle for insignificant differences: (a) autumn (SON), (b) winter (DJF), (c) spring (MAM), and (d) summer (JJA).

indices should be interpreted with care. Higher or no significant change in RMSE values for the IDW versus WRF daily $\mathrm{ET}_{0}$ forecast indicates that the low IDW biases observed in Fig. 8 were due to canceling out of positive and negative daily biases. In addition, WRF daily $\mathrm{ET}_{0}$ bias in autumn and spring was higher than in summer and winter (Fig. 6). However, since the $\mathrm{WRF}_{\mathrm{BC}}$ bias in autumn and spring was mostly positive, the smaller bias came across as a lower RMSE as well, compared to WRF (Figs. 9a,c). In winter and summer, on the other hand, WRF daily $\mathrm{ET}_{0}$ bias was generally lower, compared to spring and autumn, as was $\mathrm{WRF}_{\mathrm{BC}}$ bias, with values oscillating between negative and positive, resulting in a nonsignificantly different RMSE between WRF and $\mathrm{WRF}_{\mathrm{BC}}$ (Figs. 9b,d).

Overall, the higher IOA scores of the WRF versus IDW model means that the WRF is better able to predict variations in observed values, but its higher mean bias will result in excess irrigation over a long period of time for most of the locations examined if used for irrigation scheduling. Relying on $\mathrm{WRF}_{\mathrm{BC}}$ will reduce the excess irrigation, compared to WRF, due to significantly 
lower bias, particularly in autumn, winter and spring, for which the reduction in bias was statistically significant. Low IOA of IDW-based daily $\mathrm{ET}_{0}$ predictions means a poorer correlation with observations, whereas the low IDW bias results from mutual canceling out of positive and negative biases. This is further emphasized by the generally similar or higher RMSE in the IDW-based daily $\mathrm{ET}_{0}$ predictions compared to WRF. This type of forecast by IDW may lead to excess irrigation for some periods and deficit irrigation in others, thus, resulting in both plant stress and yield reduction, as well as overuse of water and fertilizer. Basing irrigation on the WRF Model may not be suitable as a long-range decisionmaking tool for either type of crop irrigation due to large bias. By significantly lowering the bias, $\mathrm{WRF}_{\mathrm{BC}}$ is superior to WRF and IDW methods for irrigation management, preserving the IOA of the original WRF forecast while considerably reducing bias and RMSE.

\section{Summary and conclusions}

The WRF Model was evaluated as a tool for precise irrigation in Israel with the intention of applying the derived insights to additional areas. The model's performance was examined with respect to lead time, location, season, and synoptic pressure system classification. To the best of our knowledge, this is the first work to designate WRF as a high-resolution irrigation decisionmaking tool in the Eastern Mediterranean. The WRF

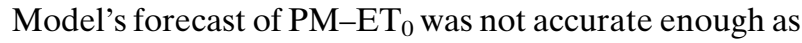
it overpredicted daily irrigation demands for most locations throughout the year by $13 \%$ on average. The $W_{s}$ bias was a major contributor to $\mathrm{ET}_{0}$ bias in this study and the bias was influenced by local features, independent of seasons and synoptic scenarios and with no geographical gradients. Therefore, measurement-based post hoc bias corrections of $W_{s}$, individually for each measurement station was beneficial in improving PM$\mathrm{ET}_{0}$ predictions, particularly for spring, winter and fall, for which the improvement was statistically significant in terms of bias.

The $\mathrm{WRF}_{\mathrm{BC}}$ correction scheme assumes a weak dependence of WRF $W_{s}$ relative bias on time and also relies on a relatively dense network of observational stations; therefore, the benefit of using the proposed hybrid approach should be further evaluated by testing these assumptions under different conditions and with different bias-correction algorithms. In our work, the $\mathrm{WRF}_{\mathrm{BC}}$ correction scheme reduced the annual mean of predicted daily $\mathrm{ET}_{0}$ bias from $13 \%$ to $3 \%$. Without applying this hybrid approach, the WRF Model was superior to the IDW interpolation method in terms of IOA but inferior in terms of model bias. Thus,
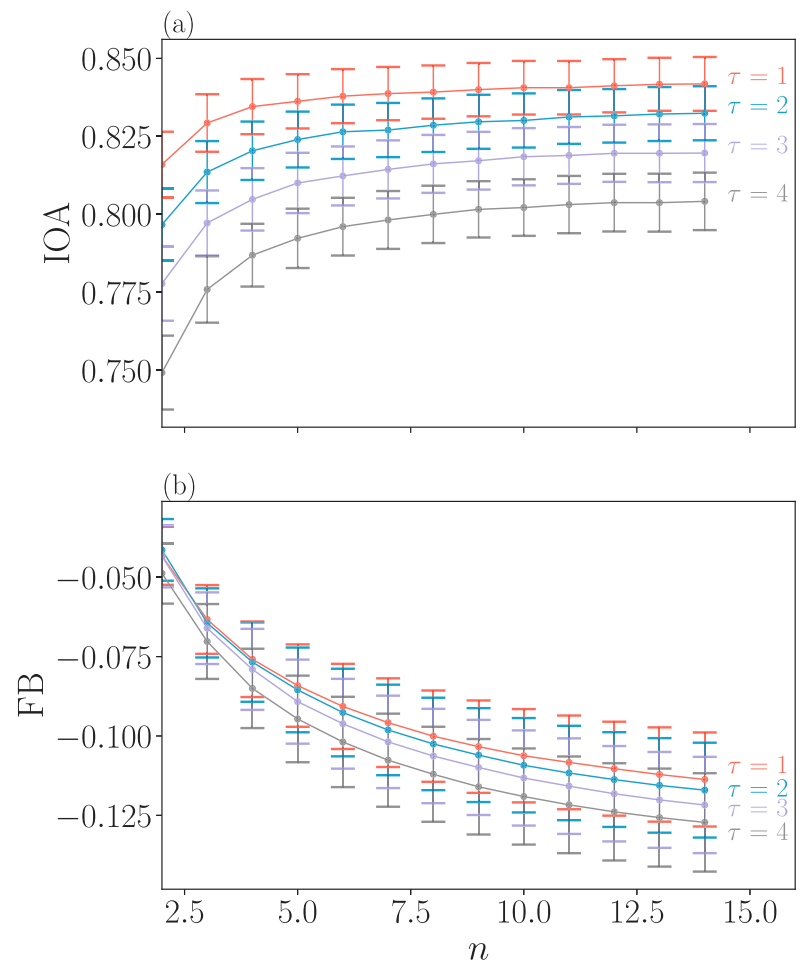

FIG. A1. (a) IOA and (b) FB of corrected wind speed $W_{s}$ with respect to the number of averaged preceding days $n$. Every point represents the mean for 29 station locations together with the standard errors. Four curves represent the 4 forecast lead days $\tau$.

forecasting $\mathrm{PM}-\mathrm{ET}_{0}$ by either past observations alone (i.e., using IDW) or by WRF with no bias correction led to a poorer outcome than that obtained by applying $W_{R F} F_{B C}$ Neither IDW nor WRF are recommended for direct use in irrigation decision-making. $\mathrm{WRF}_{\mathrm{BC}}$ had a reduced daily $\mathrm{ET}_{0}$ forecast bias, while preserving high agreement with observational trends (high IOA, meaning small additive and proportional differences between model and observations), making the WRF Model more suitable for agricultural use, which requires accurate day-to-day forecasts. In terms of precipitation, the WRF Model's ability to predict daily rain was high, which may be beneficial for agricultural practice and could potentially prevent surplus water use, fertilizer losses, and oxygen deficiency. We suggest that precipitation forecast by WRF and $\mathrm{PM}-\mathrm{ET}_{0}$ evaluation using the hybrid $\mathrm{WRF}_{\mathrm{BC}}$ can be beneficial for precise irrigation. Further study is required to quantify the benefits of this approach based on specific case studies of crop-irrigation modeling, with respect to location, crop, and irrigation method.

Acknowledgments. This research was financed by the Israeli ministry of agriculture and rural development, 
Grant 16-31-0003, and also as part of the Root of the Matter project of The Root Zone Knowledge Center for Leveraging Modern Agriculture. We are grateful to Gilad Kozokaro from "abiotix" for providing us with the WRF Model forecast results needed for this paper and also to Dr. Elad Shilo for his fruitful suggestions during the initiation of the project. Erick Fredj would like to thank Prof. Pinhas Alpert for letting us use his synoptic pressure regime classification data based on his work in Alpert et al. (2004).

\section{APPENDIX}

\section{Finding an Optimal $\boldsymbol{n}$ for the Bias Correction Scheme}

Figure A1 presents a test for the optimal averaged time window of preceding days $n$ for correcting $W_{s}$ according to the procedure described in section $2 \mathrm{e}$ and Fig. 6. The IOA and FB of the corrected WRF $W_{s}$ versus observed $W_{s}$ was calculated for a range of 2-14 for $n$ and 1-4 lead days $(\tau)$. IOA is increased with $n$ for all $\tau$ and $|\mathrm{FB}|$ also increased with $\tau$. Thus, a compromise $(n=5)$ was set to preserve high IOA while reducing FB.

\section{REFERENCES}

Ali, H., 2010: Fundamentals of Irrigation and On-Farm Water Management. Vol. 1. Springer, $560 \mathrm{pp}$.

Allen, R. G., L. S. Pereira, D. Raes, and M. Smith, 1998: Crop evapotranspiration: Guidelines for computing crop water requirements. FAO Irrigation and Drainage Paper 56, 300 pp., www.fao.org/docrep/X0490E/X0490E00.htm

Alpert, P., R. Abramsky, and B. U. Neeman, 1990: The prevailing summer synoptic system in Israel-subtropical high, not Persian trough. Isr. J. Earth Sci., 39 (2-4), 93-102.

- I. Osetinsky, B. Ziv, and H. Shafir, 2004: Semi-objective classification for daily synoptic systems: Application to the eastern Mediterranean climate change. Int. J. Climatol., 24, 1001-1011, https://doi.org/10.1002/joc.1036.

Arca, B., P. Duce, R. L. Snyder, D. Spano, and M. Fiori, 2003: Use of numerical weather forecast and time series models for predicting reference evapotranspiration. Proceedings of the Fourth International Symposium on Irrigation of Horticultural Crops, ISHS Acta Horticulturae, No. 664, International Society for Horticultural Science, 39-46.

Bauer, P., A. Thorpe, and G. Brunet, 2015: The quiet revolution of numerical weather prediction. Nature, 525, 47-55, https:// doi.org/10.1038/nature14956.

Berkovic, S., 2016: Synoptic classes as a predictor of hourly surface wind regimes: The case of the central and southern Israeli coastal plains. J. Appl. Meteor. Climatol., 55, 1533-1547, https://doi.org/10.1175/JAMC-D-16-0093.1.

Bhattarai, S. P., D. J. Midmore, and N. Su, 2010: Sustainable irrigation to balance supply of soil water, oxygen, nutrients and agro-chemicals. Biodiversity, Biofuels, Agroforestry and Conservation Agriculture, Springer, 253-286.

Breslow, N., 1970: A generalized Kruskal-Wallis test for comparing $\mathrm{K}$ samples subject to unequal patterns of censorship. Biometrika, 57, 579-594, https://doi.org/10.1093/biomet/57.3.579.
Carmona, I., and P. Alpert, 2009: Synoptic classification of moderate resolution imaging spectroradiometer aerosols over Israel. J. Geophys. Res., 114, D07208, https://doi.org/10.1029/ 2008JD010160.

Cheng, W. Y., and W. J. Steenburgh, 2005: Evaluation of surface sensible weather forecasts by the WRF and the Eta models over the western United States. Wea. Forecasting, 20, 812-821, https://doi.org/10.1175/WAF885.1.

Dayan, U., B. Ziv, A. Margalit, E. Morin, and D. Sharon, 2001: A severe autumn storm over the middle-east: Synoptic and mesoscale convection analysis. Theor. Appl. Climatol., 69, 103-122, https://doi.org/10.1007/s007040170038.

Diagne, M., M. David, J. Boland, N. Schmutz, and P. Lauret, 2014: Post-processing of solar irradiance forecasts from WRF model at reunion island. Sol. Energy, 105, 99-108, https://doi.org/ 10.1016/j.solener.2014.03.016.

Duce, P., R. Snyder, and D. Spano, 1999: Forecasting reference evapotranspiration. Proceedings of the Third International Symposium on Irrigation of Horticultural Crops, ISHS Acta Horticulturae, No. 537, International Society for Horticultural Science, 135-141.

Dudhia, J., 1989: Numerical study of convection observed during the winter monsoon experiment using a mesoscale two-dimensional model. J. Atmos. Sci., 46, 3077-3107, https://doi.org/10.1175/ 1520-0469(1989)046<3077:NSOCOD>2.0.CO;2.

Fritsch, J. M., and J. S. Kain, 1993: Convective parameterization for mesoscale models: The Fritsch-Chappell scheme. The Representation of Cumulus Convection in Numerical Models, Meteor. Monogr., No. 46, Amer. Meteor. Soc., 159-164.

García-Díez, M., J. Fernández, L. Fita, and C. Yagüe, 2013: Seasonal dependence of WRF model biases and sensitivity to PBL schemes over Europe. Quart. J. Roy. Meteor. Soc., 139, 501-514, https://doi.org/10.1002/qj.1976.

Gunwani, P., and M. Mohan, 2017: Sensitivity of WRF model estimates to various PBL parameterizations in different climatic zones over India. Atmos. Res., 194, 43-65, https://doi.org/ 10.1016/j.atmosres.2017.04.026.

Hacker, J. P., and D. L. Rife, 2007: A practical approach to sequential estimation of systematic error on near-surface mesoscale grids. Wea. Forecasting, 22, 1257-1273, https://doi.org/ 10.1175/2007WAF2006102.1.

Hanley, J. A., and B. J. McNeil, 1982: The meaning and use of the area under a receiver operating characteristic (ROC) curve. Radiology, 143, 29-36, https://doi.org/10.1148/radiology.143.1.7063747.

Hong, S.-Y., and J.-O. J. Lim, 2006: The WRF single-moment 6-class microphysics scheme (WSM6). J. Korean Meteor. Soc, 42 (2), 129-151.

Ilotoviz, E., H. Shafir, P. Gasch, and P. Alpert, 2015: Semi operational prediction of the Dead Sea evaporation-A synoptic systems approach. J. Water Resour. Prot., 7, 1058-1074, https:// doi.org/10.4236/jwarp.2015.713087.

Jiménez, P. A., and J. Dudhia, 2012: Improving the representation of resolved and unresolved topographic effects on surface wind in the WRF Model. J. Appl. Meteor. Climatol., 51, 300-316, https://doi.org/10.1175/JAMC-D-11-084.1.

Jiménez-Esteve, B., M. Udina, M. R. Soler, N. Pepin, and J. R. Miró, 2018: Land use and topography influence in a complex terrain area: A high resolution mesoscale modelling study over the eastern Pyrenees using the WRF model. Atmos. Res., 202, 49-62, https://doi.org/10.1016/j.atmosres.2017.11.012.

Jolliffe, I. T., and D. B. Stephenson, 2012: Forecast Verification: A Practitioner's Guide in Atmospheric Science. Wiley, 292 pp. 
Kioutsioukis, I., A. de Meij, H. Jakobs, E. Katragkou, J.-F. Vinuesa, and A. Kazantzidis, 2016: High resolution WRF ensemble forecasting for irrigation: Multi-variable evaluation. Atmos. Res., 167, 156-174, https://doi.org/10.1016/j.atmosres.2015.07.015.

Kool, D., A. Ben-Gal, N. Agam, J. Šimŭnek, J. Heitman, T. Sauer, and N. Lazarovitch, 2014: Spatial and diurnal below canopy evaporation in a desert vineyard: Measurements and modeling. Water Resour. Res., 50, 7035-7049, https://doi.org/10.1002/ 2014WR015409.

Kosmopoulos, P., S. Kazadzis, K. Lagouvardos, V. Kotroni, and A. Bais, 2015: Solar energy prediction and verification using operational model forecasts and ground-based solar measurements. Energy, 93, 1918-1930, https://doi.org/10.1016/ j.energy.2015.10.054.

Kumar, M., N. Raghuwanshi, and R. Singh, 2011: Artificial neural networks approach in evapotranspiration modeling: A review. Irrig. Sci., 29, 11-25, https://doi.org/10.1007/ s00271-010-0230-8.

Lara-Fanego, V., J. Ruiz-Arias, D. Pozo-Vázquez, F. SantosAlamillos, and J. Tovar-Pescador, 2012: Evaluation of the WRF model solar irradiance forecasts in Andalusia (southern Spain). Sol. Energy, 86, 2200-2217, https:// doi.org/10.1016/j.solener.2011.02.014.

Levermore, G., and J. Parkinson, 2006: Analyses and algorithms for new test reference years and design summer years for the UK. Build. Serv. Eng. Res. Tech., 27, 311-325, https://doi.org/ 10.1177/0143624406071037.

Libonati, R., I. Trigo, and C. C. DaCamara, 2008: Correction of $2 \mathrm{~m}-$ temperature forecasts using Kalman filtering technique. Atmos. Res., 87, 183-197, https://doi.org/10.1016/j.atmosres.2007.08.006.

López-Urrea, R., F. M. de Santa Olalla, C. Fabeiro, and A. Moratalla, 2006: Testing evapotranspiration equations using lysimeter observations in a semiarid climate. Agric. Water Manage., 85, 15-26, https://doi.org/10.1016/j.agwat.2006.03.014.

Luo, Y., S. Traore, X. Lyu, W. Wang, Y. Wang, Y. Xie, X. Jiao, and G. Fipps, 2015: Medium range daily reference evapotranspiration forecasting by using ANN and public weather forecasts. Water Resour. Manage., 29, 3863-3876, https://doi.org/10.1007/ s11269-015-1033-8.

Mann, H. B., and D. R. Whitney, 1947: On a test of whether one of two random variables is stochastically larger than the other. Ann. Math. Stat., 18, 50-60, https://doi.org/10.1214/aoms/1177730491.

Mlawer, E. J., S. J. Taubman, P. D. Brown, M. J. Iacono, and S. A. Clough, 1997: Radiative transfer for inhomogeneous atmospheres: RRTM, a validated correlated-k model for the longwave. J. Geophys. Res., 102, 16 663-16 682, https://doi.org/ 10.1029/97JD00237.

Noble, E., L. M. Druyan, and M. Fulakeza, 2014: The sensitivity of WRF daily summertime simulations over West Africa to alternative parameterizations. Part I: African wave circulation. Mon. Wea. Rev., 142, 1588-1608, https://doi.org/10.1175/ MWR-D-13-00194.1.

_, — , and — 2017: The sensitivity of WRF daily summertime simulations over West Africa to alternative parameterizations. Part II: Precipitation. Mon. Wea. Rev., 145, 215-233, https:// doi.org/10.1175/MWR-D-15-0294.1.
Perera, K. C., A. W. Western, B. Nawarathna, and B. George, 2014: Forecasting daily reference evapotranspiration for Australia using numerical weather prediction outputs. Agric. For. Meteor., 194, 50-63, https://doi.org/10.1016/j.agrformet.2014.03.014.

Piper, B., 1989: Sensitivity of penman estimates of evaporation to errors in input data. Agric. Water Manage., 15, 279-300, https://doi.org/10.1016/0378-3774(89)90021-8.

Powers, J. G., and Coauthors, 2017: The weather research and forecasting model: Overview, system efforts, and future directions. Bull. Amer. Meteor. Soc., 98, 1717-1737, https://doi.org/ 10.1175/BAMS-D-15-00308.1.

Ries, H., and K. H. Schlünzen, 2009: Evaluation of a mesoscale model with different surface parameterizations and vertical resolutions for the Bay of Valencia. Mon. Wea. Rev., 137, 2646-2661, https://doi.org/10.1175/2009MWR2836.1.

Ruiz, J. J., C. Saulo, and J. Nogués-Paegle, 2010: WRF Model sensitivity to choice of parameterization over South America: Validation against surface variables. Mon. Wea. Rev., 138, 3342-3355, https://doi.org/10.1175/2010MWR3358.1.

Santos-Alamillos, F., D. Pozo-Vázquez, J. Ruiz-Arias, V. LaraFanego, and J. Tovar-Pescador, 2013: Analysis of WRF model wind estimate sensitivity to physics parameterization choice and terrain representation in Andalusia (southern Spain). J. Appl. Meteor. Climatol., 52, 1592-1609, https://doi.org/ 10.1175/JAMC-D-12-0204.1.

Shepard, D., 1968: A two-dimensional interpolation function for irregularly-spaced data. Proc. 1968 23rd ACM National Conf., ACM, New York, NY, 517-524, https://doi.org/10.1145/ 800186.810616.

Siegal, S., 1956: Nonparametric Statistics for the Behavioral Sciences. McGraw-Hill, 399 pp.

Skamarock, W., and Coauthors, 2008: A description of the Advanced Research WRF version 3. NCAR Tech. Note NCAR/ TN-475+STR, 113 pp., https://doi.org/10.5065/D68S4MVH.

Srivastava, P. K., T. Islam, M. Gupta, G. Petropoulos, and Q. Dai, 2015: WRF dynamical downscaling and bias correction schemes for NCEP estimated hydro-meteorological variables. Water Resour. Manage., 29, 2267-2284, https:// doi.org/10.1007/s11269-015-0940-z.

Steduto, P., T. C. Hsiao, E. Fereres, and D. Raes, 2012: Crop yield response to water. FAO Irrigation and Drainage Paper 66, 500 pp., http://www.fao.org/3/i2800e/i2800e.pdf.

Wang, D., and X. Cai, 2009: Irrigation scheduling-Role of weather forecasting and farmers' behavior. J. Water Resour. Plann. Manage., 135, 364-372, https://doi.org/10.1061/(ASCE) 0733-9496(2009)135:5(364).

Wang, H., X.-Y. Huang, D. Xu, and J. Liu, 2014: A scale-dependent blending scheme for WRFDA: Impact on regional weather forecasting. Geosci. Model Dev., 7, 1819-1828, https://doi.org/ 10.5194/gmd-7-1819-2014.

Willmott, C. J., 1981: On the validation of models. Phys. Geogr., 2, 184-194, https://doi.org/10.1080/02723646.1981.10642213.

Zittis, G., P. Hadjinicolaou, and J. Lelieveld, 2014: Comparison of WRF Model physics parameterizations over the MENACORDEX domain. Amer. J. Climate Change, 3, 490-511, https://doi.org/10.4236/ajcc.2014.35042. 